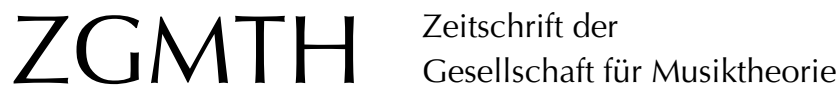

Kunkel, Liselotte (2012): Akkordstrukturen in George Gershwins Porgy and Bess. ZGMTH 9/1, 113-143. https://doi.org/10.31751/658

(C) 2012 Liselotte Kunkel

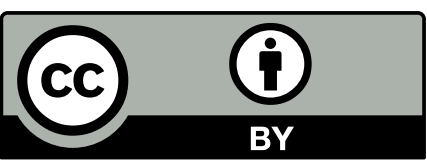

Dieser Text erscheint im Open Access und ist lizenziert unter einer Creative Commons Namensnennung 4.0 International Lizenz.

This is an open access article licensed under a

Creative Commons Attribution 4.0 International License.

veröffentlicht / first published: 26/11/2012

zuletzt geändert / last updated: 18/11/2013 


\title{
Akkordstrukturen in George Gershwins Porgy and Bess
}

\author{
Liselotte Kunkel
}

ASTRACT: Die farbige Jazz-Harmonik George Gershwins beruht auf einem charakteristischen Akkordrepertoire. Anhand seiner Oper Porgy and Bess soll dieses Repertoire systematisch dargestellt und für die Unterrichtspraxis fruchtbar gemacht werden.

\section{MEHRKLÄNGE MIT DOMINANTISCHER SEPTAKKORDBASIS}

Der weitaus größte Anteil der von Gershwin gebrauchten Akkorde lässt sich auf eine dominantische Septakkordbasis nach dem Modell: `Grundton - große Terz - reine Quinte - kleine Septime z zurückführen (die Quinte kann, wie noch zu zeigen sein wird, unter bestimmten Umständen fehlen). Hinzu kommen Optionstöne, die die Terzenschichtung weiterführen, also die große oder kleine None, die übermäßige Undezime und die große oder kleine Tredezime. Diese Optionstöne erscheinen entweder einzeln oder in unterschiedlichen Kombinationen über der Septakkordbasis. Im Folgenden werden die häufigsten Konstellationen erläutert.

\subsection{Akustische Mehrklänge}

Der Name der so genannten akustischen Skala leitet sich von der Naturtonreihe ab. Die akustische Skala entspricht den Naturtönen 8 bis 14, die in eine gleichstufig temperierte Stimmung übertragen werden. Sie lässt sich entweder als Mixolydisch mit lydischer (übermäßiger) Quarte oder als Lydisch mit mixolydischer (kleiner) Septime verstehen.

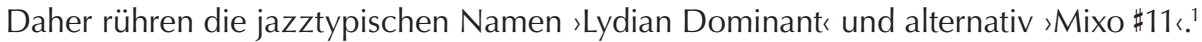

Im folgenden Beispiel sind in den (angedeuteten) Tönen der Singstimme und dem ersten Akkord der Klavierbegleitung alle Töne einer C-akustischen Skala vertreten, nämlich die Töne $c$ - $d$-e-fis-g-a-b. ${ }^{2}$

1 Vgl. Gárdonyi/Nordhoff 2002, 173.

2 Aus praktischen Gründen sind die Beispiele dem Klavierauszug (siehe Notenverzeichnis) entnommen, der allerdings keine Taktzahlen enthält. Die Notenbeispiele werden daher mit Seitenzahl und Nummer des Systems angegeben; die Taktzählung erfolgt in jedem Beispiel von 1 bis x. Die Angabe der Akkordnamen folgt der jazztypischen englischen Symbolik (z. B. Ebmin statt es-Moll, F \#7 statt Fis7). 


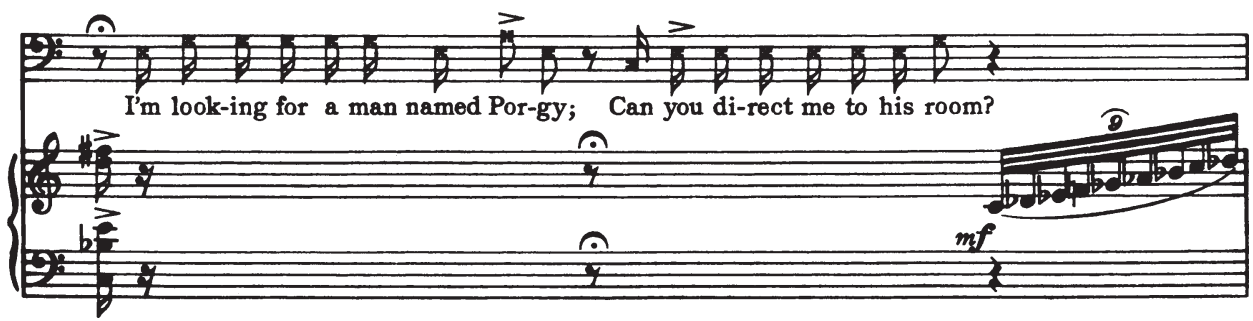

Beispiel 1: George Gershwin, Porgy and Bess, Akt 2, Szene 1 (»Dey's a Buckra comin' ...»), 229/4

Auch Beispiel 2 enthält sämtliche Töne der C-akustischen Skala (T. 3, 2. Halbe, bis T. 4; der Ton gis ist lediglich figurativ).
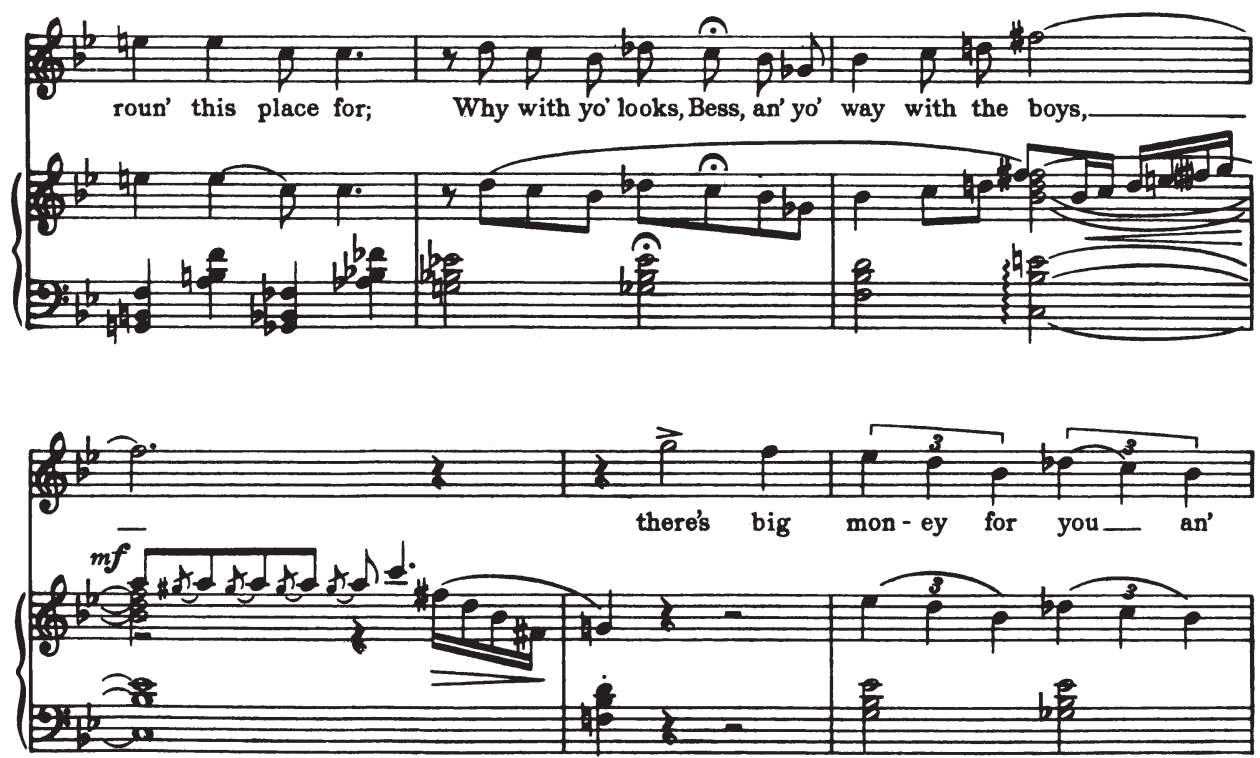

Beispiel 2: George Gershwin, Porgy and Bess, Akt 2, Szene 1 (»Picnic is alright...«), 245/3 f.

In beiden Beispielen scheint es angemessener, von einem sakustischen statt von einem sdominantischen Mehrklang، zu sprechen, da keine Auflösung in einen tonikalen F-DurKlang erfolgt. (In Beispiel 1 steht nach der aufsteigenden Tonleiter des letzten Viertels ein es-Moll-Dreiklang.) Beispiel 3 hingegen zeigt in Takt 2 einen (diesmal unfigurierten) akustischen Mehrklang auf dem Grundton c (mit den Optionstönen große None, übermäßige Undezime und große Tredezime), der sich erwartungsgemäß in die Zwischentonika F-Dur auflöst. Zwar ließe sich hier mit größerem Recht von einem >dominantischen « Mehrklang reden, doch scheint es klarer, die Bezeichnung der Klanggestalt als rakustisch von der funktionalen Bestimmung als >Dominante` grundsätzlich zu trennen. 


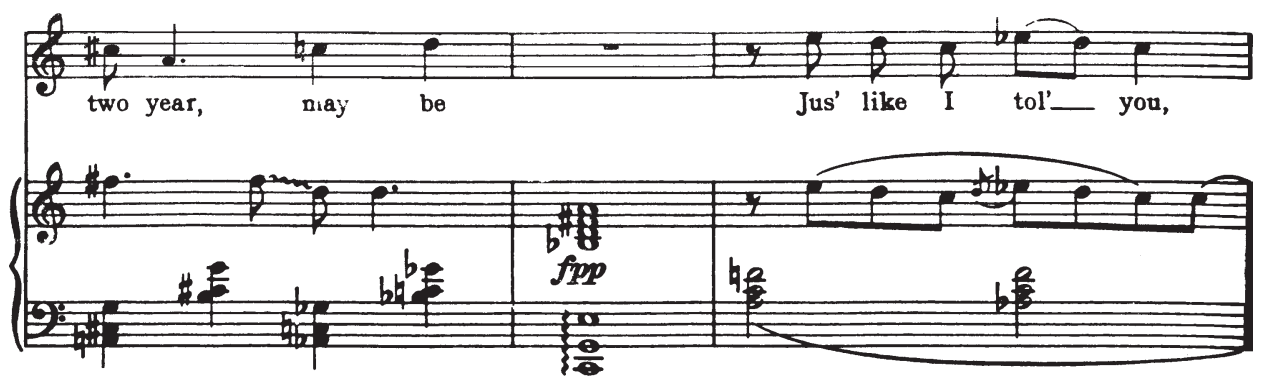

Beispiel 3: George Gershwin, Porgy and Bess, Akt 3, Szene 2 (»Sister, that Porgy ...«), 494/3

Sehr oft finden sich akustische Mehrklänge, die nicht alle sieben Töne der akustischen Skala enthalten, sondern nur einen Teil davon. Gemeinsam ist ihnen die charakteristische Basis aus Durdreiklang und kleiner (akustischer) Septime. Die große None ist der weitaus häufigste Optionston. Mit der Hinzufügung weiterer Optionstöne steigt der Schärfegrad, der sich von der Entfernung der jeweiligen Töne zum Akkordgrundton (gemessen in reinen Quinten) her bestimmen lässt.

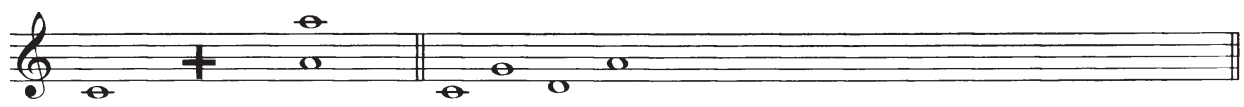

Hinzufügung ersten Grades: große Sexte (bzw. Tredezime). Entfernung vom Grundton in der Anzahl reiner Quinten in gleicher Richtung: +3 .

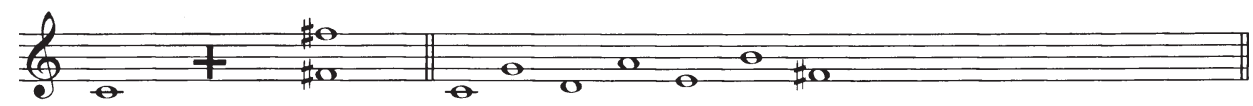

Hinzufügung zweiten Grades: Tritonus (bzw. übermäßige Undezime). Quint-Entfernung vom Grundton: +6 .

Beispiel 4: Darstellung der Quintentfernungen in Gárdonyi/Nordhoff 1990, 170

\section{Akustische Nonakkorde}

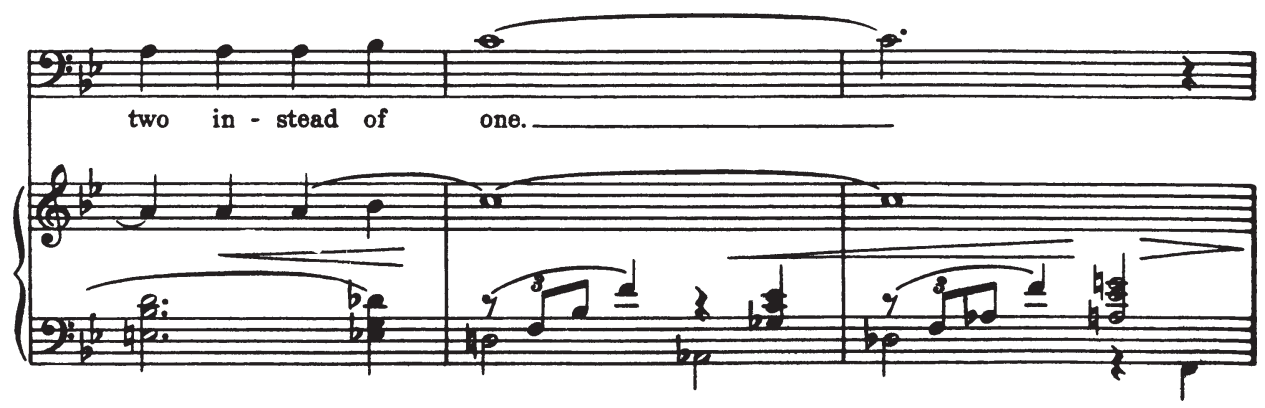

Beispiel 5: George Gershwin, Porgy and Bess, Akt 2, Szene 1 (»Bess, you is my woman now«), 252/4 
Akustische Nonakkorde ohne weiteren Optionston kommen zuweilen in einem einfacheren harmonischem Umfeld vor und fungieren in der Regel als Dominanten. In Beispiel 5 (vorige Seite) finden sich vor F7/9 (T. 3, 2. Halbe) zwei Vierklänge (Ab7 in T. 2, 2. Halbe, und D bmaj7 in T. 3, 1. Halbe). Es folgt ein tonikaler Klang über dem Ton $b$ (im Beispiel nicht wiedergegeben).

In Beispiel 6 steht Bb7/9 (T. 2) im Kontext einfacher Vier- und sogar Dreiklänge.

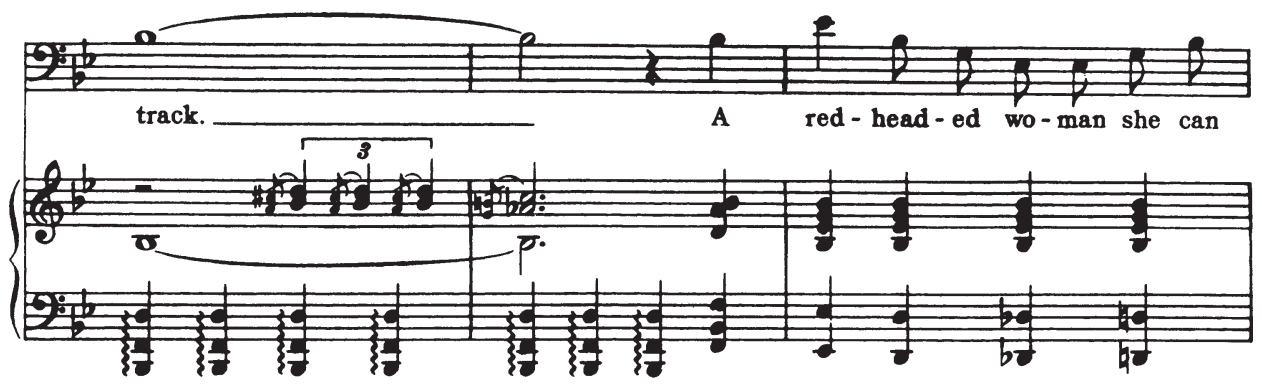

Beispiel 6: George Gershwin, Porgy and Bess, Akt 2, Szene 4 (»A redheaded woman«), 421/1

In beiden Beispielen liegt direkt über dem Grundton ein primärer Akkordton, nämlich die Terz (Beispiel 5, T. 3, 1. Halbe) oder die Quinte (Beispiel 6). Dieser stabile Klangaufbau ist im Jazz-Klaviersatz am gebräuchlichsten. ${ }^{3}$ In Beispiel 7 hingegen trägt der Nonakkord über dem Grundton $f$ (T. 1, 2. Halbe) als nächsthöheren Ton die None und wirkt daher labiler.

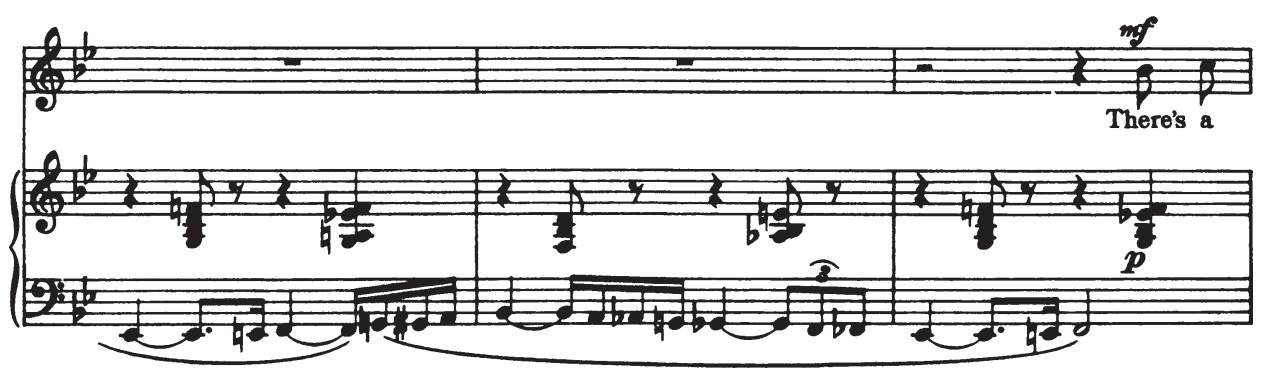

Beispiel 7: George Gershwin, Porgy and Bess, Akt 3, Szene 2 (»There's a boat dat's leavin'«), 497/2

Anhand des Voicings von Beispiel 8 kann die sogenannte `drop-2«-Technik erläutert werden. ${ }^{4}$ Würde man über dem Basston $d$ (T. 2, 4. Viertel) alle anderen Akkordtöne in engster Quintlage spielen, so ergäbe sich ein sehr gängiges Voicing, in dem über dem Grundton als nächsthöherer Ton die Septime liegen würde. Wenn man nun den zweit-

3 Bezugspunkt ist hier stets der Klavierauszug, der von den Voicings in der Originalpartitur abweichen kann.

4 Zum Begriff `drop 2«vgl. Levine 1992, 171. 
höchsten Ton dieses engen Voicings um eine Oktave nach unten `droppt`, dann ergibt das ein ebenfalls gutklingendes Voicing in gemischter Lage mit der Terz direkt über dem Grundton. (Die direkt vorausgehenden Akkorde zeigen weitere gängige Akkordhinzufügungen und Lagen und werden später kommentiert.)

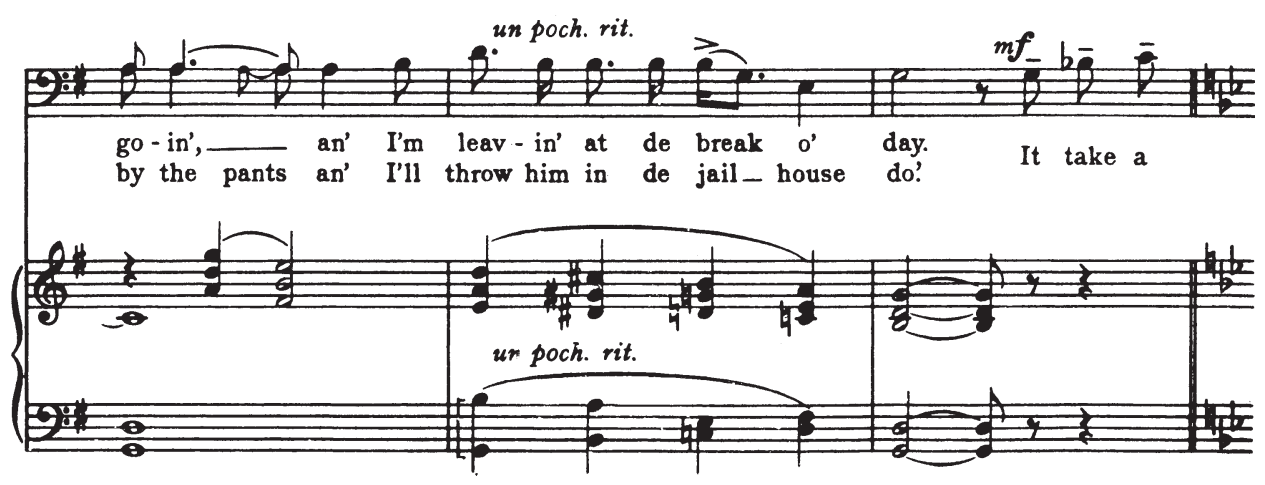

Beispiel 8: George Gershwin, Porgy and Bess, Akt 2, Szene 1 (»Oh, I'm agoin` out«), 190/3

\section{Akustische Tredezimakkorde}

Akustische Tredezimakkorde können als Basis einen Septakkord mit oder ohne None haben. Ersteres ist bei Gershwin allerdings weit häufiger. Beispiel 9 zeigt zunächst über dem Grundton $f$ (T. 2, 1. Halbe) die Ausprägung mit None (hier enharmonisch entstellt als fisis) und direkt danach über dem Grundton $h$ den Klang ohne None.

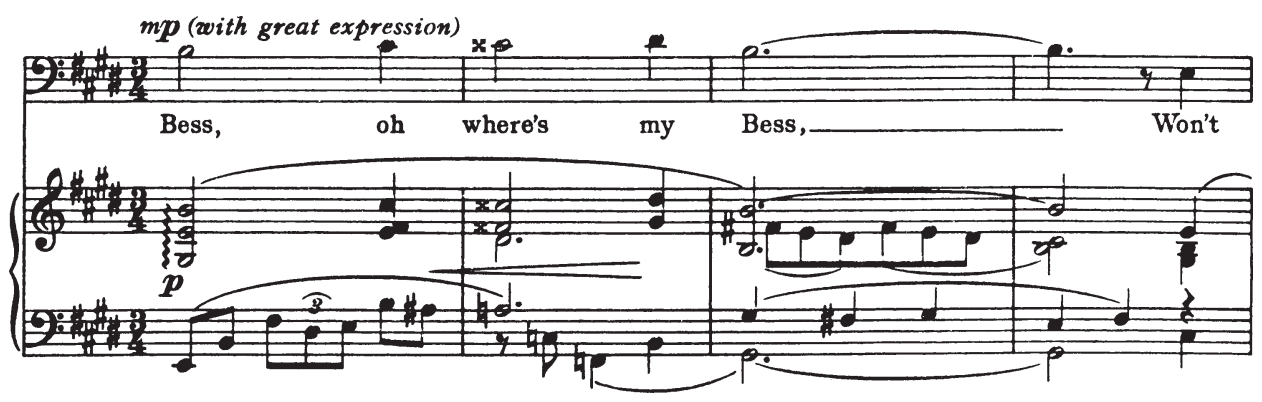

Beispiel 9: George Gershwin, Porgy and Bess, Akt 3, Szene 3 (»Bess, oh where's my Bess«), 538/3

Ferner kann die Septakkordbasis entweder quinthaltig oder quintlos sein. Im vorliegenden Stück treten viele Tredezimakkorde einschließlich der Quinte auf; im Jazz-Klaviersatz wird die Quinte aus klanglichen (Reibung mit der Tredezime in enger Lage) und grifftechnischen Gründen ( zu wenige Finger`) oft weggelassen. Im vorangegangenen Beispiel 9 ist der erste Tredezimakkord quinthaltig (Ton c auf der 2. Achtel), der zweite quintlos. 
Beispiel 10 zeigt in der rechten Hand (T. 1) ein sehr typisches Klaviervoicing für akustische Tredezimakkorde. Es wird umgangssprachlich auch als Großsekundvoicing bezeichnet, da die beiden mittleren Töne $d$ und $e$ in der engen Tredezimlage (Tredezime als höchster Ton) eine große Sekunde bilden. Ebenso typisch wäre das Kleinsekundvoicing, das sich in der engen Nonenlage ergibt; die beiden mittleren Töne $a$ und $b$ bilden dann eine kleine Sekunde. Beide Voicings, wirken über dem Grundton sehr stabil: Beim Großsekundvoicing liegt, falls man ohne Quinte spielt, direkt über dem Grundton die Septime, beim Kleinsekundvoicing hingegen die Terz, also jeweils einer der beiden übrigen Haupttöne des Klangs. Der Akkord in Beispiel 10 wird zusätzlich durch den Quintton $g$ der linken Hand stabilisiert und wirkt eher `derb`.

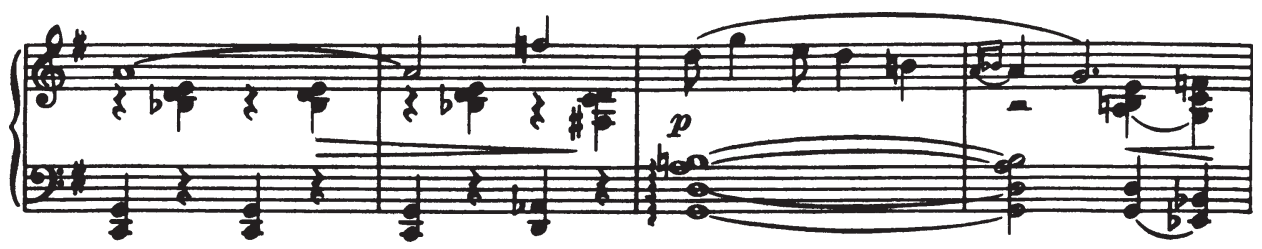

Beispiel 10: George Gershwin, Porgy and Bess, 3. Akt, 3. Szene, Vorspiel 508/5

In Beispiel 8 (s.o.) findet sich ein Tredezimakkord (T. 2, 2. Viertel), der wiederum als ‘drop-2-voicing، aufgetragen ist: Stünden alle Töne über dem Grundton $h$ in engster Lage, ergäbe sich das erwähnte Kleinsekundvoicing. Wird bei diesem engen Voicing der zweite Ton von oben dann um eine Oktave 'gedroppt‘, erhält man die gut klingende gemischte Lage des Beispiels.

Vom Tredezimakkord klar zu unterscheiden ist ein weiterer Akkordtyp, der ebenfalls die große Sexte über dem Grundton enthält: Da den beiden letzten Akkorden in Beispiel 10 die Septakkordbasis fehlt, werden sie nicht als Tredezimakkorde bezeichnet, sondern wahlweise als Durdreiklang mit zugefügter Sexte und None (abgekürzt G6/9 bzw. Eb6/9) oder als Do-Pentatonik.

\section{Akustische Undezimakkorde}

Akustische Undezimakkorde können als Basis einen Septakkord mit oder ohne None haben; Letzteres ist allerdings selten. Der Undezimakkord erinnert, wenn die None fehlt, an den übermäßigen Terzquartakkord der Wiener Klassik, zumal wenn er sich in der Auflösung als doppelleittönig zum Grundton einer nachfolgenden Dominante wie in Beispiel 11 erweist (T. 1, 4. Viertel, und Folgeakkord).

Die doppelleittönige Auflösung zum Grundton einer nachfolgenden Tonika wie in Beispiel 12 (T. 2, 4. Viertel, und Folgeakkord) erinnert hinsichtlich der Akkordverbindung an entsprechende Stellen etwa bei Franz Schubert.

Die Quinte kann fehlen; hier gelten ähnliche Kriterien wie beim Tredezimakkord (klangliche Reibungen; grifftechnische Möglichkeiten im Klaviersatz). Da die dissonante Option der übermäßigen Undezime oft weit oben im Klang liegt (wie etwa auch im 


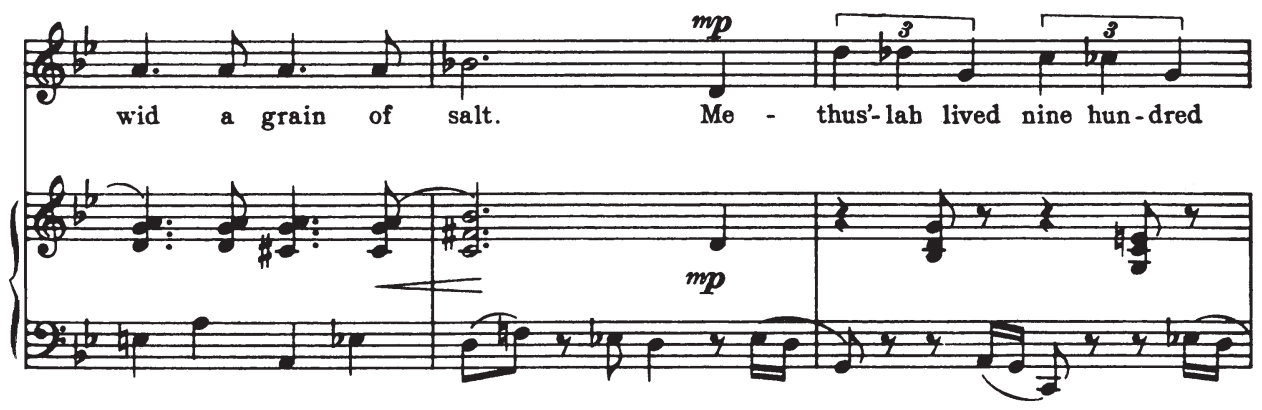

Beispiel 11: George Gershwin, Porgy and Bess, Akt 2, Szene 2 (»lt ain't necessarily so«), 293/2

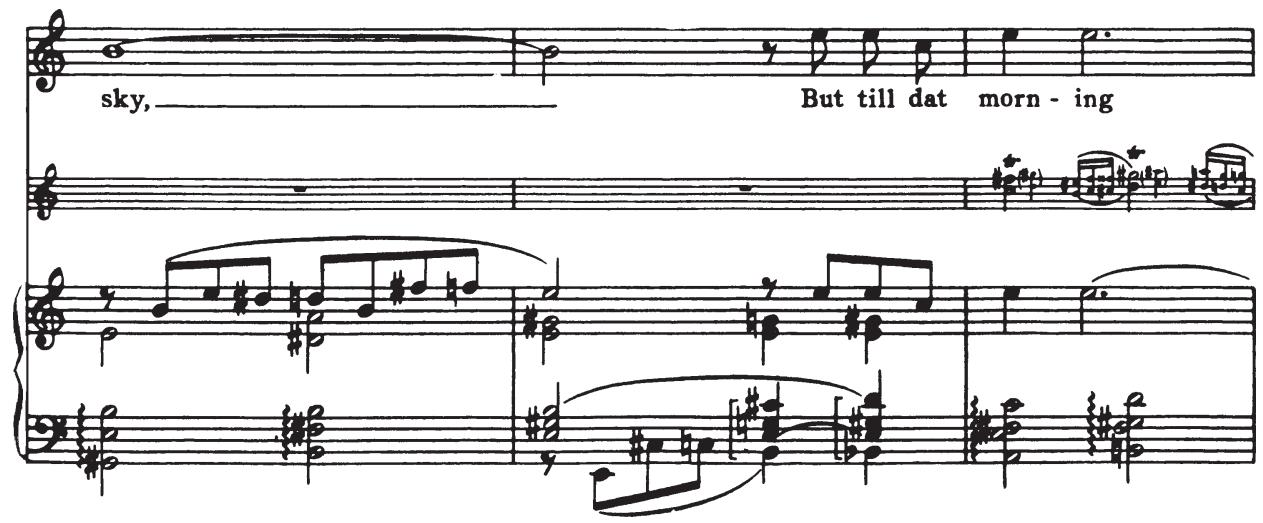

Beispiel 12: George Gershwin, Porgy and Bess, Akt 2, Szene 4 (»One of dese mornings«), 389/3

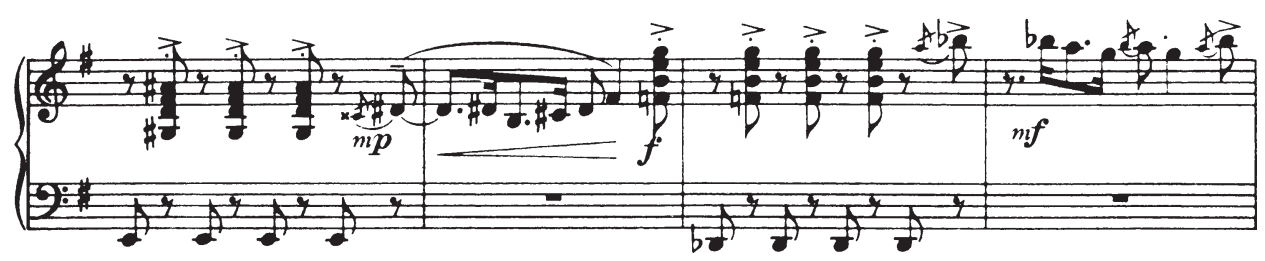

Beispiel 13: George Gershwin, Porgy and Bess, Akt 1, Szene I, Vorspiel, 5/3

eröffnenden Klavierakkord von Beispiel 1), repräsentiert Beispiel 13 (T. 1) das am ehesten realisierbare Voicing in der engen Lage dar. (Über dem Grundton des steht ebenfalls ein Klang mit $\sharp 11$; er enthält aber zusätzlich die $\sharp 9$, ist also kein akustischer Klang und wird später kommentiert.)

Demgegenüber zeigt Beispiel 14 (T. 2) die sderbere` quinthaltige Ausprägung des Akkords. 


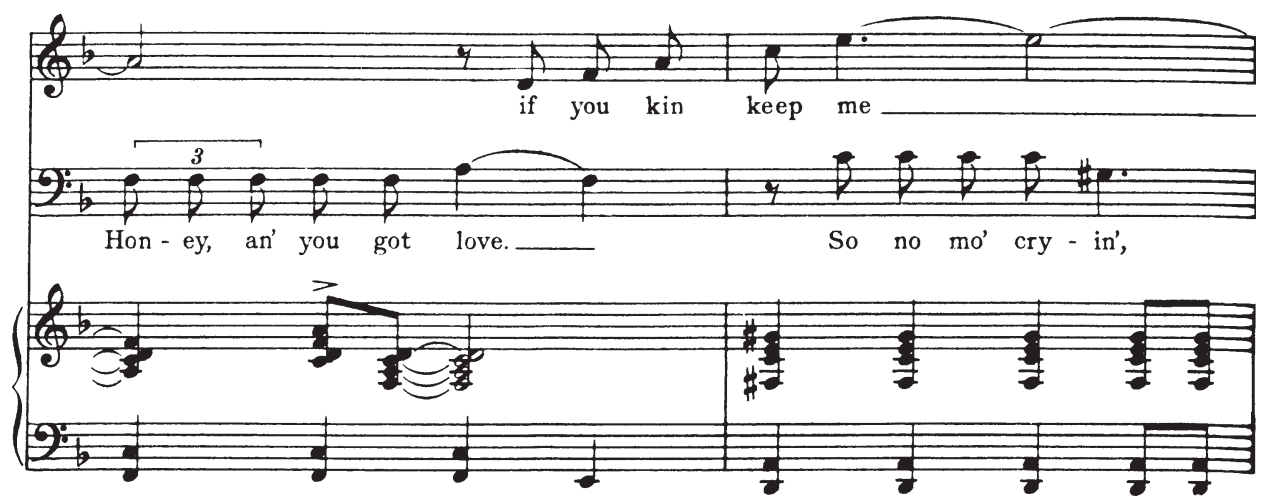

Beispiel 14: George Gershwin, Porgy and Bess, Akt 2, Szene 3 (»l loves you, Porgy«), 356/2

Ferner können Undezimakkorde entweder mit oder ohne Tredezime auftreten. Beispiel 15 ermöglicht den klanglichen Vergleich: Im Klaviersatz (T. 1) steht ein akustischer Undezimakkord über es, die Singstimme wechselt zwischen der Tredezime $c$ und dem Grundton es.

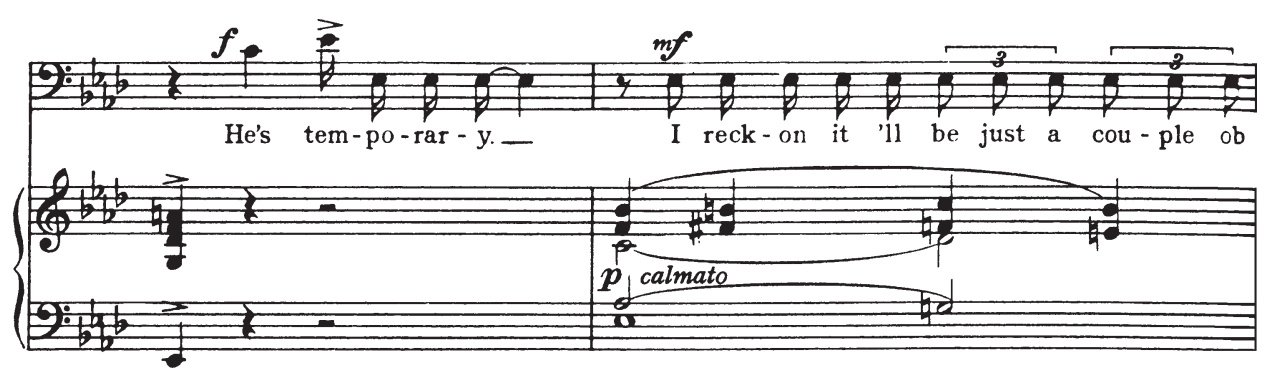

Beispiel 15: George Gershwin, Porgy and Bess, Akt 2, Szene 2 (»You sho’ got funny tas` in men«), $308 / 3$

\subsection{Alterierte Mehrklänge}

Der Name salteriert oder englisch saltered hang auf die so genannte alterierte Skala oder saltered scale`, die eine Parallelskala der akustischen Skala darstellt: Nimmt man den vierten Skalenton (Tritonus über dem akustischen Grundton) der akustischen Skala als neuen Grundton an, so erhält man die alterierte Skala. Beispiel 16 zeigt dies anhand der Skalen C-Akustisch und F\#-altered. ${ }^{5}$

5 Vgl. ebd., 175. 


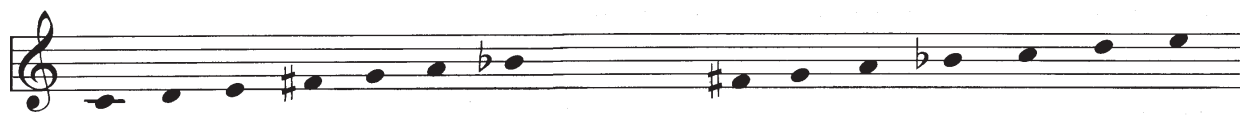

Beispiel 16: Alterierte Skala als Parallelskala der akustischen Skala

Der Name lässt sich auf die beiden Möglichkeiten beziehen, die Skala abzuleiten: Der ersten Erklärung zu Folge gleicht die alterierte Skala einer Durskala mit hochalteriertem Grundton: Erschiene in Beispiel 16 nicht fis, sondern $f$, handelte es sich um eine F-DurSkala. Diese Erklärung folgt in ihrer Logik denjenigen Benennungen, die für die anderen Parallelskalen der akustischen Skala üblich sind: Sie beziehen sich ebenfalls auf diatonische Modi (meist Kirchentonarten) und deren Abweichungen, beispielsweise Mixo b6.

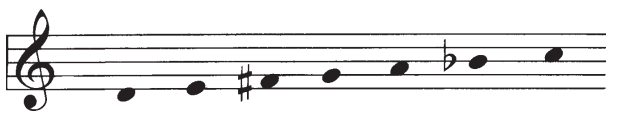

Beispiel 17: Mixo b6

Die zweite Erklärung besagt, dass in der alterierten Skala außer den eigentlichen Funktionstönen, also der großen 3 und der kleinen 7, alle Töne alteriert sind. So finden sich über dem Grundton (hier: fis) die folgenden Intervalle: b9, \#9 bzw. b10, \#11 und b13 bzw. $\# 5$. $^{6}$

Sowohl die Ableitung als auch die Verwendung der alterierten Skala sind eng verknüpft mit dem Prinzip der Tritonussubstitution: Anstelle eines Akkords mit akustischem Basis-Septakkord oder im Wechsel mit ihm kann der Akkord über dem tritonusentfernten Grundton verwendet werden. Die Funktionstöne (große Terz und kleine Septime) bilden in beiden Akkorden einen enharmonisch klanggleichen Tritonus und erfüllen jeweils die umgekehrte Funktion: Die Terz des einen Akkordes ist die Septime des anderen und umgekehrt.7 In Beispiel 9 kann man diesen Sachverhalt nachvollziehen: Über dem Grundton $f$ (T. 2, 2. Viertel) stehen der Terzton a und der bereits als dis notierte Septimton (eigentlich es). Über dem nachfolgenden Grundton $h$ fungiert umgekehrt a als Septime und dis als Terz. Hier handelt es sich um eine sukzessive Tritonussubstitution: Die beiden tritonusentfernten Akkorde erklingen nacheinander.

Im Falle einer simultanen Tritonussubstitution erklingt der Grundton des ersten Akkords zusammen mit den akustischen Optionstönen des tritonusentfernten Akkords, so dass ein Akkord der alterierten Skala entsteht. (Übertragen auf die Situation in Beispiel 16 hieße das: Über dem Grundton fis erklingen die Optionstöne aus der C-akustischen Skala.)

Ferner kann man von einer alternativen Tritonussubstitution sprechen: Dabei erklingt nicht der erwartete Akkord (etwa die $\mathrm{V}$ in einer II-V-I-Kadenz), sondern nur deren Tritonusvertreter. Es entsteht eine II-bII-I-Kadenz, wobei die bll akustisch oder alteriert besetzt sein kann.

6 Vgl. ebd., 74.

7 vgl. ebd., $45 \mathrm{f}$. 
Bei Gershwin finden sich alterierte Klänge sowohl in Form der sukzessiven als auch der simultanen Tritonussubstitution. Beispiel 18 beginnt als stilisiertes Kinderlied mit dem Akkordpendel I-V-I (T. 1-3). Der dominantische Septakkord über $h$ (T. 2, 2. Viertel) ist besetzt mit der großen 13 (Ton gis). Vorgeschaltet ist diesem Klang der tritonusentfernte Grundton $f$. Über ihm bildet der Ton gis die $¥ 9$, die als zusätzliche kleine Terz (zur bereits vorhandenen großen Terz a) gehört werden kann. (Im klassischen Jazz ist die Reihenfolge der Akkorde oft eine andere: Zuerst erklingt der erwartete Akkord - hier die V. Stufe - danach der tritonusentfernte Klang.)

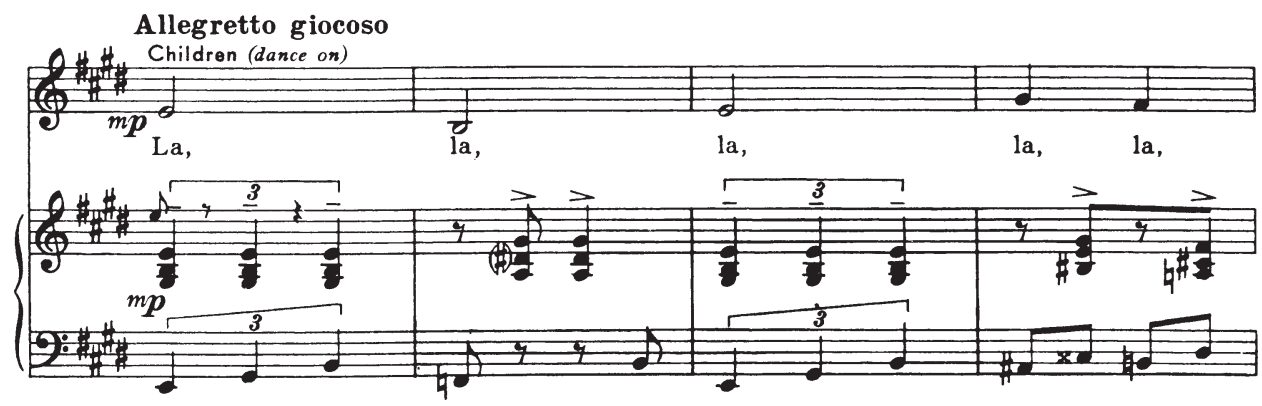

Beispiel 18: George Gershwin, Porgy and Bess, Akt 3, Szene 3 (»La, la, la«), 515/2

Beispiel 19 zeigt über dem Grundton $d$ einen der bereits besprochenen akustischen Mehrklänge mit großer None und großer Tredezime (T. 2, 4. Viertel). Ein Viertel zuvor erklingen die gleichen Töne über dem tritonusentfernten Grundton as, wobei ein Klang mit $\# 9$ (Ton $h$ ) und $\# 5$ (Ton e) entsteht. Hier handelt es sich also ebenso wie im vorigen Beispiel um eine sukzessive Tritonussubstitution.

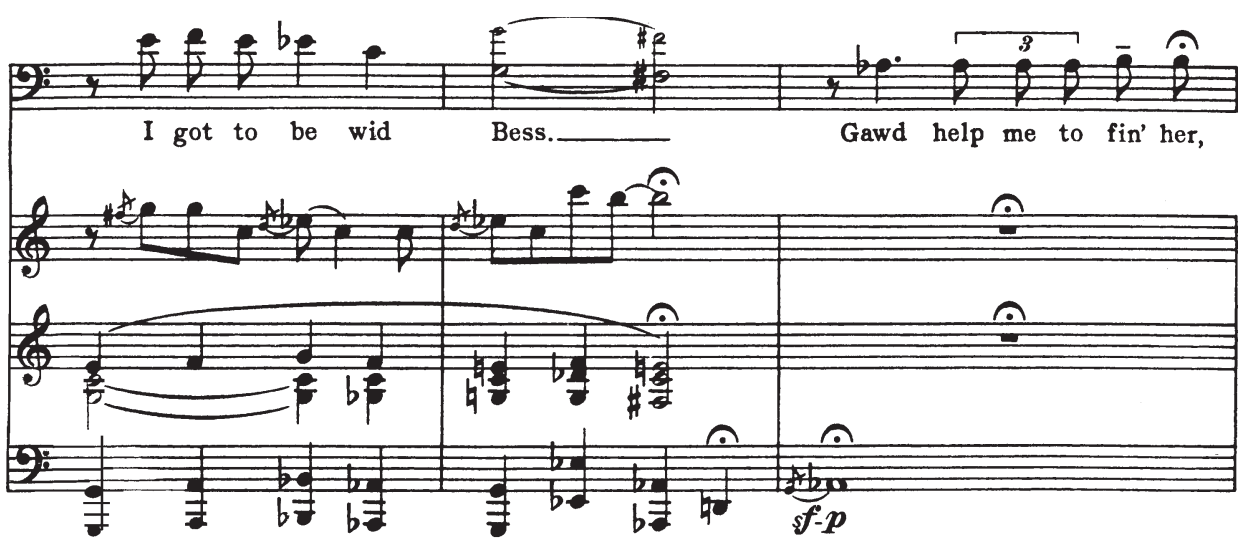

Beispiel 19: George Gershwin, Porgy and Bess, Akt 3, Szene 3 (»Ain't you say ...«), 554/2 
Klänge mit simultaner Tritonussubstitution können so notiert sein, dass man die beiden tritonusentfernten `Teilklänge` deutlich erkennt. Beispiel 20 zeigt über der Basis D7 einen As-Dur-Dreiklang (T. 1); daraus resultiert insgesamt ein alterierter Klang über $d$ mit b9und $\# 11$, letztere notiert als b5. Beispiel 21 (T. 2) zeigt über dem Grundton a den Klang Eb7; daraus resultiert ein alterierter Klang über a, ebenfalls mit b9 und \#11 (notiert als b5). Die Gestaltung der Singstimme als Es-Dur-Dreiklangsbrechung verstärkt den Eindruck einer Übereinanderlagerung zweier Klänge über verschiedenen Grundtönen.

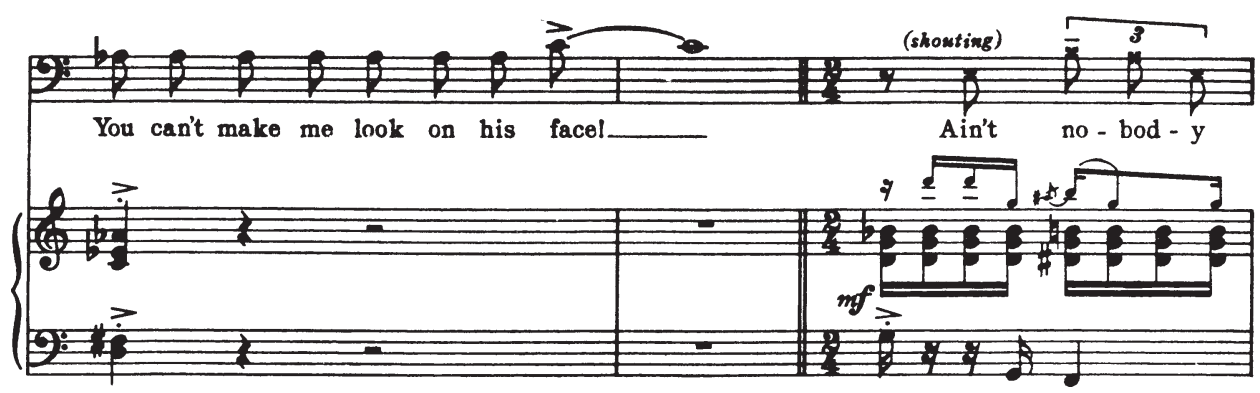

Beispiel 20: George Gershwin, Porgy and Bess, Akt 3, Szene 2 (»Turn me loose«), 492/1

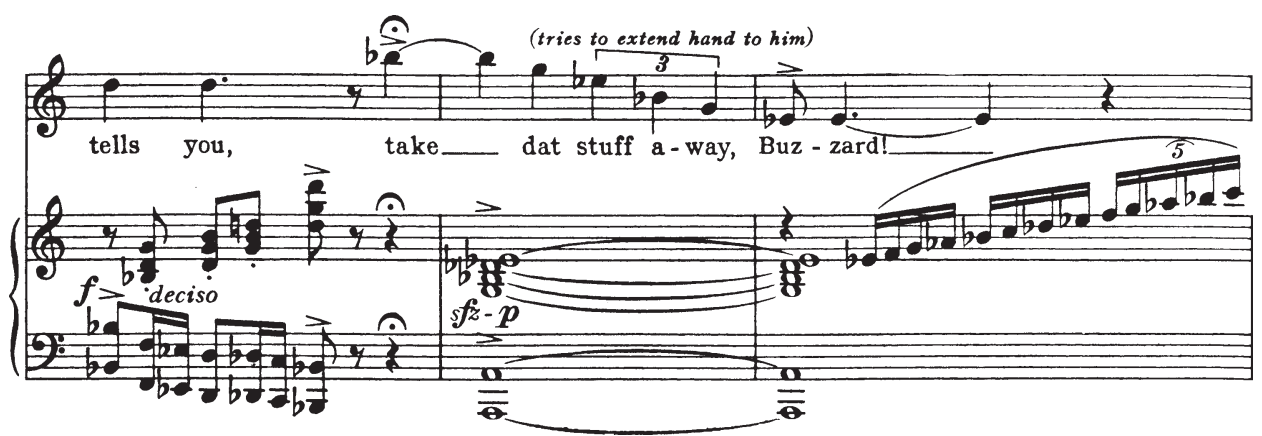

Beispiel 21: George Gershwin, Porgy and Bess, Akt 3, Szene 2 (»Sister, that Porgy ain' goin' ...«), $496 / 2$

Die alterierten Mehrklänge mit b9, $\# 9$ (alias b10) und $\sharp 5$ alias b13 oder einer Auswahl aus diesen Optionstönen können aber auch so notiert sein, dass über dem Grundton kein tritonusentfernter Mehrklang zu erkennen ist. Auch der tritonusentfernte Grundton selbst (also die \#11) kann fehlen.

Nur selten erscheinen alle drei der genannten Optionstöne gleichzeitig in einem Akkord, möglicherweise wegen des hohen Dissonanzgrades. Gelegentlich findet man sie in figurierter Form wie im oben gezeigten Beispiel 11: Nach der sukzessiven Tritonussubstitution im 1. Takt des Systems erklingt D7/b13. Der Bass fügt dann in figurativer Linie noch die b10 (Ton $f$ ) und die b9 (Ton es) hinzu. Der Dissonanzgrad der hinzugefügten Töne kommt dadurch, dass sie nacheinander erklingen, weniger stark zum Tragen. 
Ebenso erscheint in Beispiel 22 über dem Basisakkord A7/b13 eine kurze figurative Linie aus b10 und b9 (T. 2, 2. Halbe), was an eine spätromantische Idiomatik erinnert. In beiden Beispielen sind je 6 von 7 Tönen der alterierten Skala vertreten; die $\sharp 11$ über dem Grundton fehlt.

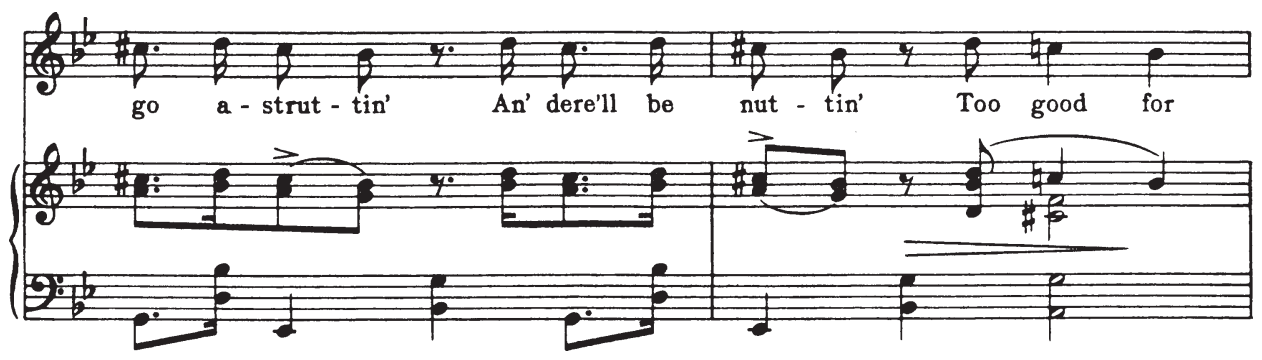

Beispiel 22: George Gershwin, Porgy and Bess, Akt 3, Szene 2 (»There's a boat dat's leavin' soon «), 499/2

Verschiedene Kombinationen aus den Optionstönen sind hingegen oft zu finden. Beispiel 23 zeigt über dem Grundton cis (T. 3, 1. Halbe) einen Klang mit b9 (Ton $d$ in der Singstimme) sowie b10 (Ton e im Klaviersatz). Allerdings lässt sich auch hier noch eine (unvollständige und teilweise enharmonisch entstellte) Kombination aus C\#7 und G7 erkennen, wofür vor allem der Ton $g$, also die als b5 notierte $\# 11$ verantwortlich zeichnet.

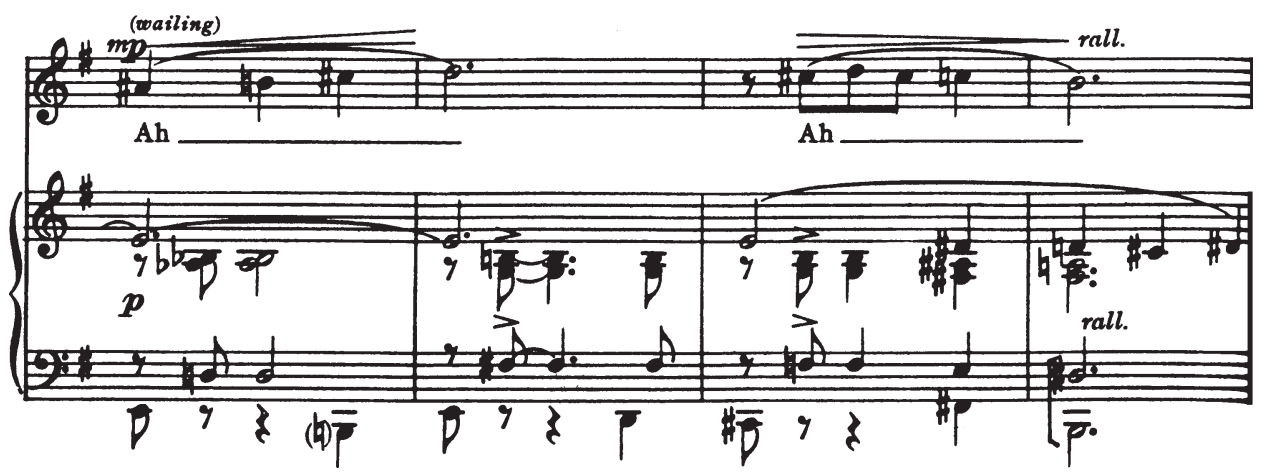

Beispiel 23: George Gershwin, Porgy and Bess, Akt 1, Szene 2 (»My man's gone now«), 154/4

Beispiel 24 zeigt in T. 3 die häufige Kombination aus b9 (Ton des) und b13 (Ton as). Die Bassbrechung vom Grundton c zur $\# 11$ bzw. b5 ges lässt Gb7, also wiederum den tritonusentfernten Akkord anklingen.

Beide Konstellationen (b9 mit b10 sowie b9 mit b13) kommen aber an vielen Stellen auch ohne die $\# 11$ vor. Ebenso finden sich unzählige Akkorde mit nur einem einzigen Optionston über dem Basisakkord aus Grundton, Terz und Septime (also z. B. D7/b9 oder D7/b10 oder D7/b13); eine detaillierte Aufschlüsselung (wie sie bei den akustischen Mehrklängen vorgenommen wurde) erübrigt sich an dieser Stelle. 


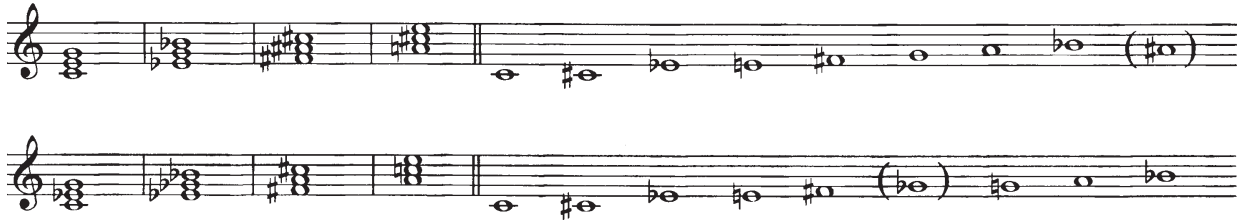

Beispiel 24: George Gershwin, Porgy and Bess, Akt 3, Szene 2 (»There's a boat dat's leavin' soon «), 497/4

\subsection{Distanzielle Mehrklänge}

Distanzielle Mehrklänge beruhen auf einer Aufteilung der Oktave in gleichgroße Schritte. Für die vorliegenden Betrachtungen sind dabei zwei Arten relevant, nämlich die kleinterzige und die ganztönige Aufteilung.

\section{Kleinterzige Aufteilung der Oktave}

Werden Mehrklänge auf Grundtönen gebildet, die kleinterzig voneinander entfernt sind, und diese Mehrklänge dann kombiniert, entstehen neue Mehrklänge, die in bestimmten Konstellationen für Gershwins Harmonik charakteristisch sind. Für den einfacheren JazzKlaviersatz sind sie weniger wichtig als die akustischen und alterierten Klänge, dennoch sollen sie, anknüpfend an das bereits Gesagte, kurz aufgeführt und erläutert werden.

Die zugrundeliegende Skala ist bei allen folgenden Klängen die Halbton-Ganztonskala (beginnend mit einem Halbton über dem Grundton), auch alternierende Achtstufigkeit genannt. Ihr Tonvorrat ergibt sich aus der Tonsumme aller Dur- oder Molldreiklänge im Kleinterzabstand (Beispiel 25). ${ }^{8}$ In dieser Skala können akustische Septakkorde mit den Optionstönen der b9, der b10, der $\# 11$ und der großen 13 entstehen. Der Unterschied zu den alterierten Mehrklängen besteht also im möglichen Erscheinen der reinen Quinte (die in der alterierten Skala nicht enthalten ist) und der großen 13 anstelle der b13.

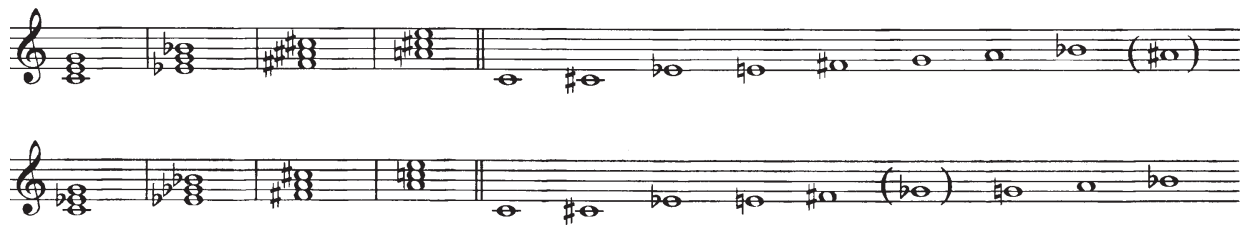

Beispiel 25: Alle Dur- bzw. Molldreiklänge einer Oktave im Kleinterzabstand und die resultierende Skala ${ }^{9}$

Sowohl die Halbton-Ganztonskala als auch deren Parallelskala, nämlich die GanztonHalbtonskala (beginnend mit einem Ganzton über dem Grundton), sind im Jazz sehr

8 Vgl. Gárdonyi/Nordhoff 2002, 220.

9 Ebd. 
gebräuchlich und werden üblicherweise als `diminished scales` oder ıverminderte Skalen bezeichnet. $^{10}$

Wie der Name andeutet, enthält die verminderte Skala die typischen Töne, mit denen beispielsweise über verminderte Septakkorde improvisiert wird. Beispiel 26 zeigt diejenige Skala, mit der man über $\mathrm{C} \# \mathrm{O} 7$ improvisieren würde.

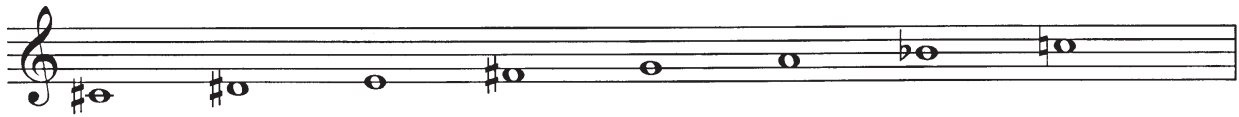

Beispiel 26: Diminished scale

Die kleinterzige Struktur ist bei Gershwin in manchen Klängen deutlich sichtbar. Beispiel 27 zeigt über dem Grundton e (T. 1, 4. Viertel) einen Septakkord (Dur-Dreiklang mit kleiner Septime) mit den Hinzufügungen der b10 (Ton g) und der \#11 (Ton $b$, also notiert als b5). Notiert ist er so, dass die linke Hand einen E-Dur-Dreiklang, die rechte Hand dagegen einen g-Moll-Dreiklang spielt. Ähnlich verhält es sich in Takt 3 des Beispiels: Hier werden ein C-Dur-Dreiklang und ein Es-Dur-Dreiklang zu einem C7/b10-Klang (1. Halbe) sowie ein D-Dur-Dreiklang und ein F-Dur-Dreiklang zu einem D7/b10-Klang (2. Halbe) kombiniert.

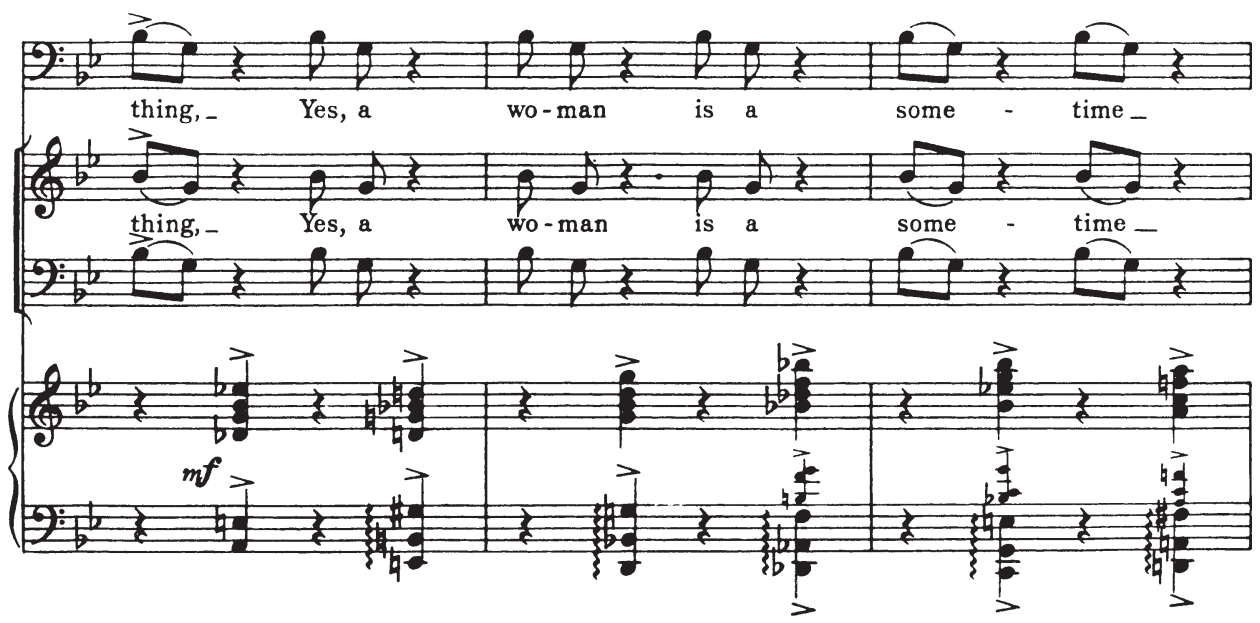

Beispiel 27: George Gershwin, Porgy and Bess, Akt 1, Szene 1 (»Oh, a woman is a sometime thing "), 39/3

In Beispiel 28 spielt die linke Hand einen C-Dur-Dreiklang, die rechte Hand hingegen einen Ges-Dur-Dreiklang (T. 2). Daraus ergibt sich der häufig vorkommende 7/b9/\#11-Klang (\#11 als b5 notiert).

10 Vgl. Levine 1992, 78. 


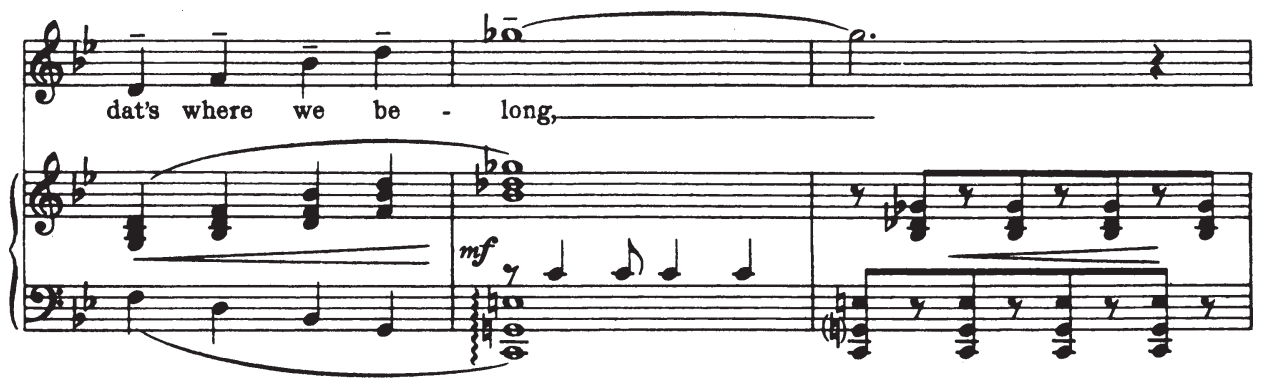

Beispiel 28: George Gershwin, Porgy and Bess, Akt 3, Szene 2 (»There's a boat dat's leavin' soon«), 501/2

Besonders gut lässt sich die distanzielle Struktur in Beispiel 29 erkennen (T. 3). Notiert ist in der linken Hand ein D-Dur-Dreiklang; nachschlagend dazu ein f-Moll-Dreiklang. Die rechte Hand spielt einen As-Dur-Dreiklang. Nimmt man alle Töne pedalisierend zusammen, hört man den Klang D 7/b9/\#11. Auch hier ist die $\# 11$ wieder als b5 notiert.

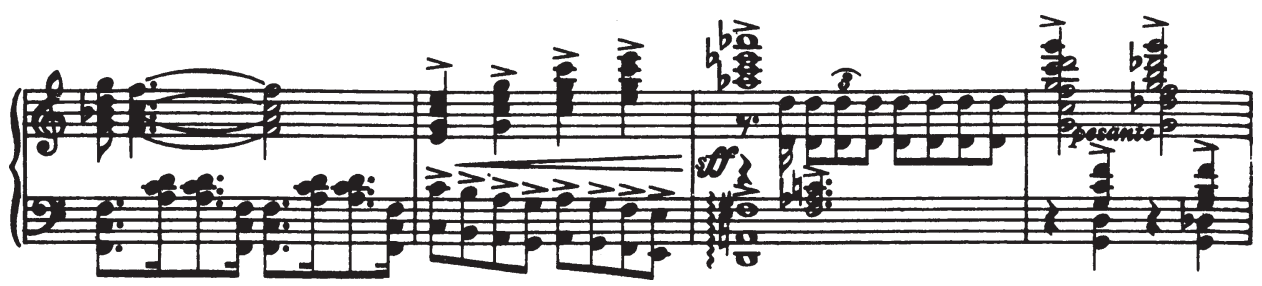

Beispiel 29: George Gershwin, Porgy and Bess, Akt 3, Ende von Szene 2, 504/5

Unterschiedliche Kombinationen der verschiedenen Optionstöne sind denkbar. Neben den besonders gebräuchlichen Typen 7/b10 und 7/b9/\#11 findet sich wie in Beispiel 30 oft der b9/13-Klang (T. 4). Auch hier ist deutlich die kleinterzige Struktur aus einem C7Akkord und einem A-Dur-Dreiklang erkennbar. Die b9 ist daher als $\sharp 8$ notiert.

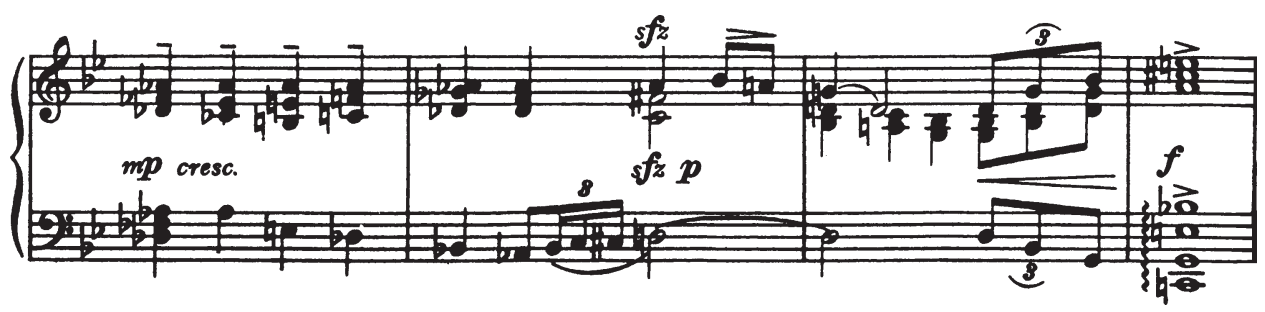

Beispiel 30: George Gershwin, Porgy and Bess, Akt 1, Szene 2 (»Gone, gone, gone«), 114 (ohne Chorstimmen)

Beispiel 31 zeigt in der linken Hand einen Doppelterzklang über G (System 1 und 2). Die rechte Hand figuriert den Akkord Gmin7. Die später hinzutretende Überstimme spielt 
dazu eine reale Sequenz und figuriert den kleinterzig entfernten Klang B bmin7. Dadurch treten zu dem Doppelterzklang noch die $\# 11$ (notiert als b5) und die b9 hinzu. Im dritten System ändert sich der Klangtypus der linken Hand (jeweils 1. Klang des 1. und 2. Taktes): Über dem neuen Grundton $b$ hört man nunmehr einen 7/b10/13-Klang.
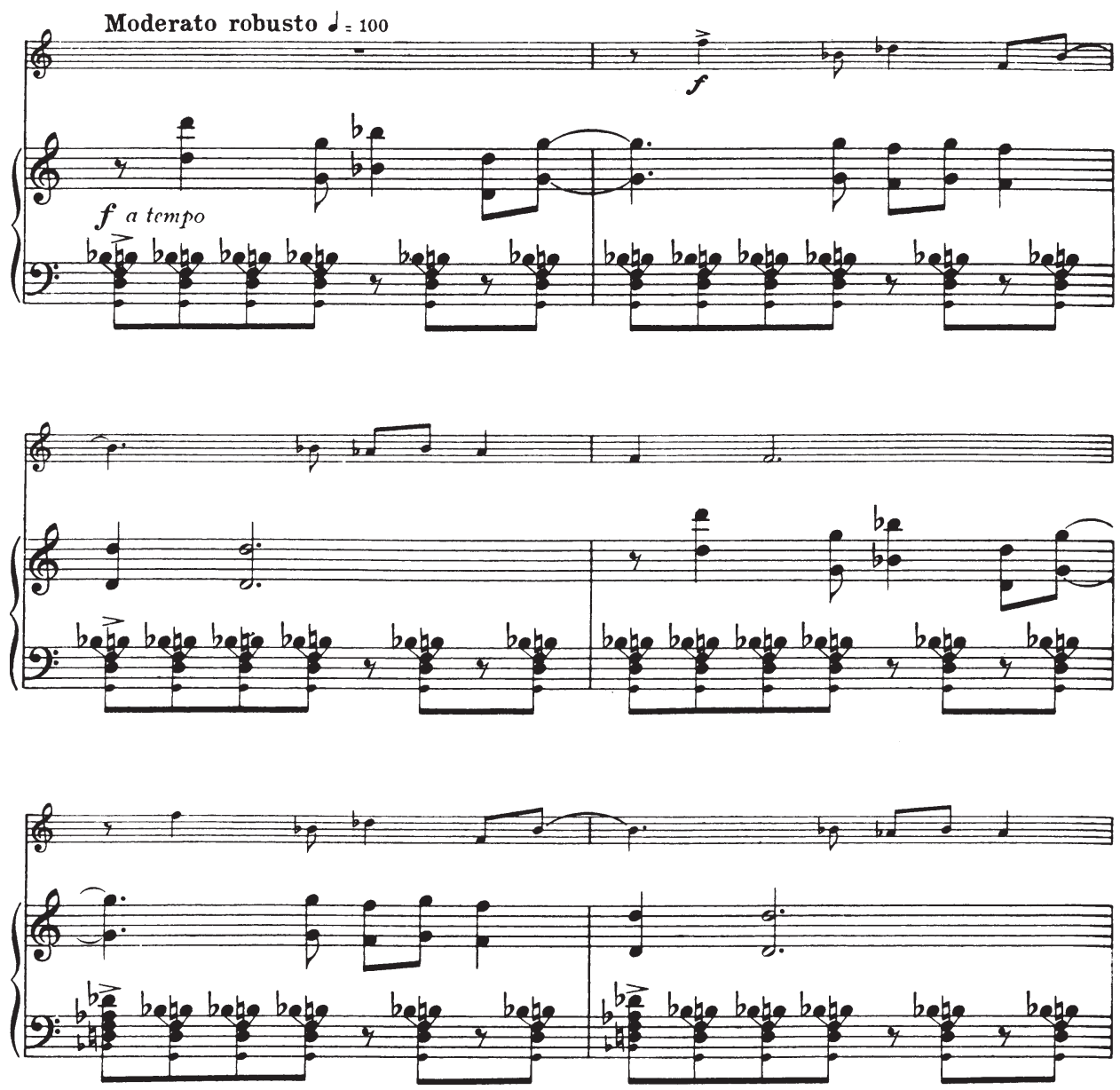

Beispiel 31: George Gershwin, Porgy and Bess, Akt 2, Szene 2 (»Take yo' hands off me«), 320/1 ff.

Ein völlig anderer Klangtyp, der ebenfalls auf der alternierenden Achtstufigkeit beruht, soll hier erwähnt werden, obwohl er bei weitem nicht so oft vorkommt wie die bereits beschriebenen Klänge. Beispiel 32 zeigt in realer Mixtur einen Fünfklang, der in der Bartók-Forschung als Gamma-Akkord bezeichnet wird. Er kann als Ausschnitt einer alternierenden Achtstufigkeit betrachtet werden, die in Form von zwei verminderten Septakkorden aufgetragen ist. Eine typische Ausprägung im Tastensatz ist dabei diejenige, bei der die beiden Daumen eine große Sekunde voneinander entfernt sind. In der Bartók- 
Forschung wird diese Gestalt des alternierend achtstufigen Totals als Alpha-Akkord bezeichnet. ${ }^{11}$

Der vollständige Alpha-Akkord zum ersten im Beispiel wiedergegebenen GammaAkkord (Töne $d$-f-gis-ais-cis) besteht aus den beiden verminderten Septakkorden mit den Tönen $h$ - $d$-f-gis und ais-cis-e-g (wobei enharmonische Umnotierungen üblich sind).

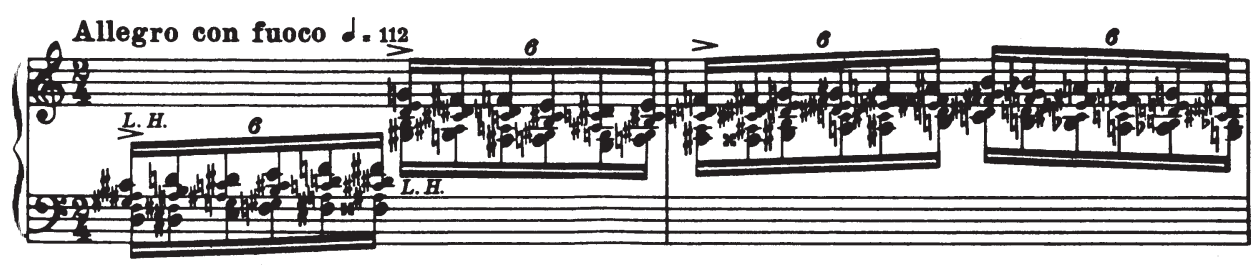

Beispiel 32: George Gershwin, Porgy and Bess, Akt 2, Szene 4 (»Oh, dere's somebody knockin' at de do'«), 405/1

Auch in den Mittelstimmen des C 7/b9/13-Klanges (die b9 ist notiert als \#8) in Beispiel 33 befindet sich der Ausschnitt eines Alpha-Akkords mit der typischen großen Sekunde (Töne $b$-cis-e-g-a-c; vollständig hieße der Alpha-Akkord b-cis-e-g und darüber a-c-esges).

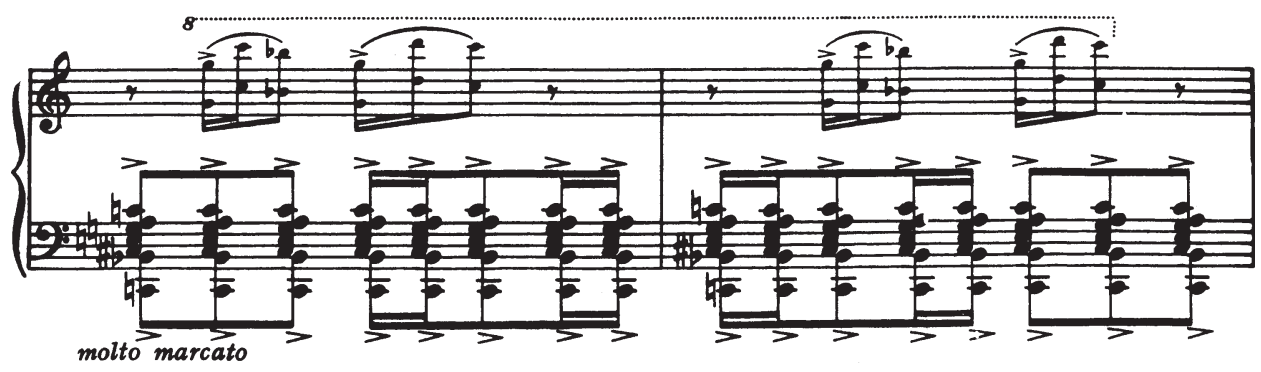

Beispiel 33: George Gershwin, Porgy and Bess, Akt 2, Szene 4 (»Oh, dere's somebody knockin' at de do' $\left.^{\prime}\right), 396 / 3$

\section{Ganztönige Aufteilung der Oktave}

In den genannten Klängen kommen fast alle Kombinationsmöglichkeiten der Optionstöne vor: große und kleine None, kleine Dezime bzw. übermäßige None, übermäßige Undezime und große oder kleine Tredezime. Dabei treten große und kleine None alternativ und nicht gleichzeitig auf. Ebenso verhält es sich mit den beiden Qualitäten der Tredezime. (Die kleine None wird gelegentlich mit der übermäßigen None kombiniert, wohingegen bei Gershwin (und allgemein im klassischen Jazz) die Kombination große plus übermäßige None nicht vorkommt.)

11 Vgl. Gárdonyi/Nordhoff 2002, 211 . 
Nicht erwähnt wurde bisher der recht seltene Klangtyp 7/9/b13. Er lässt sich auf eine ganztönige Skala zurückführen. In Beispiel 34 (T. 1, 3. Viertel) enthielte die vollständige, zum Klang passende Ganztonskala folgende Töne: $d$-e-fis-gis-b-c.
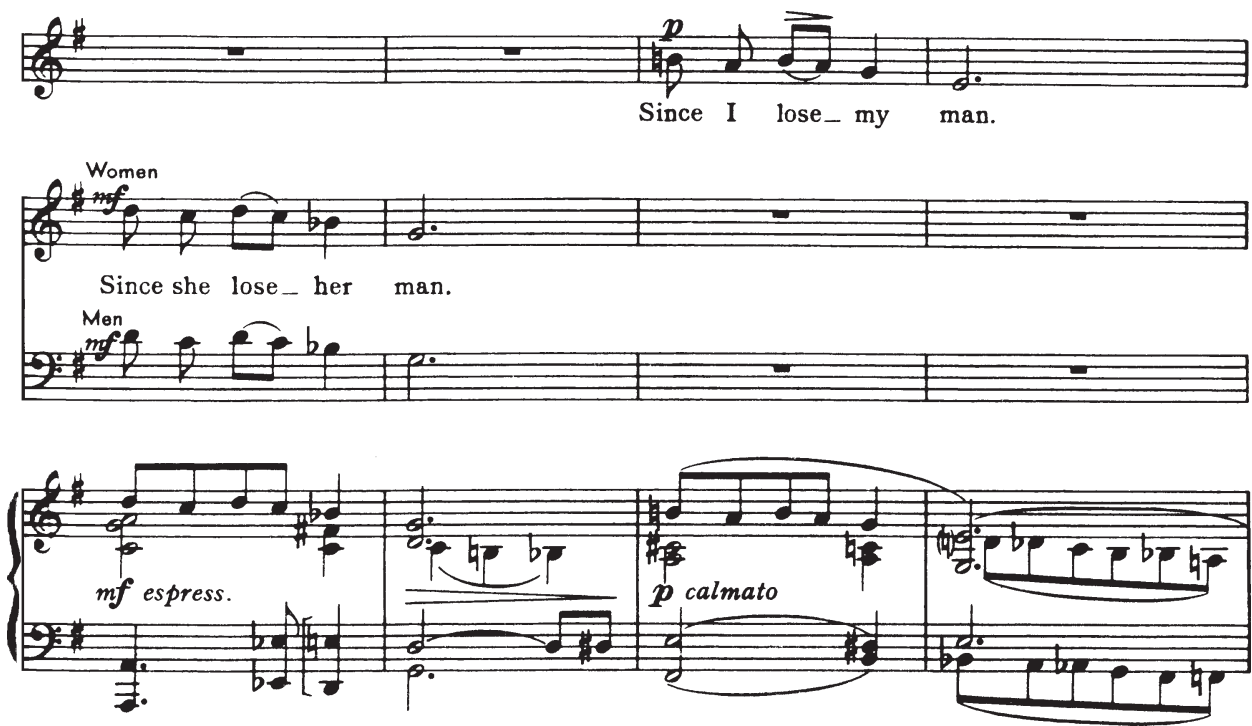

Beispiel 34: George Gershwin, Porgy and Bess, Akt 1, Szene 2 (»My man's gone now«), 157/1

Auch die (noch seltenere) Hinzufügung der \#11 zu diesem Akkordtyp geht in der ganztönigen Skala auf. Der Klang über as in Beispiel 35 (T. 2) enthält alle Töne der Ganztonskala as-b-c- $d$-e-fis. Der Ton $d$ ist dabei die $\sharp 11$.

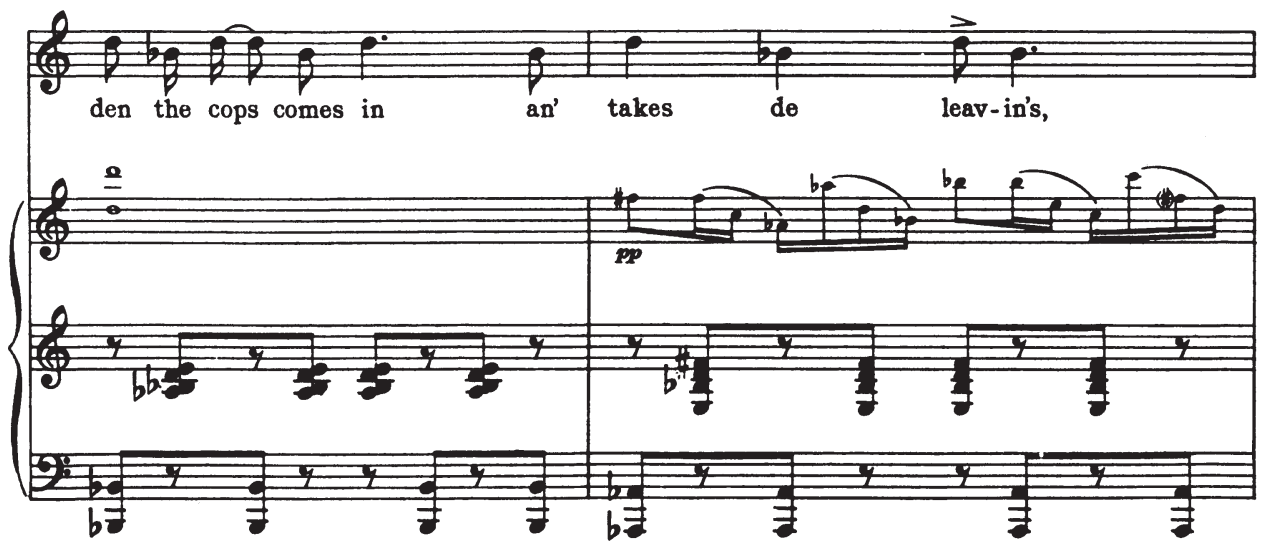

Beispiel 35: George Gershwin, Porgy and Bess, Akt 3, Szene 1 (»Crown, Crown«), 457/3 


\section{MEHRKLÄNGE MIT MAJOR-SEPTAKKORD-BASIS}

Mehrklänge mit einer Basis aus Durdreiklang und großer Septime (so genannte MajorSeptakkorde) zeigen nur wenige Möglichkeiten der Erweiterung. Sie werden weitaus seltener gebraucht als die Klänge mit akustischer Septakkordbasis. Oft stehen sie auf der I. oder IV. Stufe einer funktionalen Akkordverbindung (etwa im Rahmen von Kadenzen). Beispiel 8 (s.o.) zeigt einen Major-Septakkord mit der sehr häufigen Hinzufügung der großen None über dem Grundton c, der aktuellen IV. Stufe (T. 2, 3. Viertel).

Beispiel 7 enthält ebenfalls auf der IV. Stufe einen Major-Nonakkord in einem sehr gängigen Voicing, nämlich der engen Nonlage (T. 1, 3. Achtel).

Anhand der gemischten Nonlage von Beispiel 36 (T. 3, ohne die kleingedruckten Überstimmen) kann die `drop-3 «-Technik gezeigt werden: Der Klang über $f$ entsteht, indem man aus der engen Nonlage heraus den dritten Ton von oben um eine Oktave nach unten `droppt`.

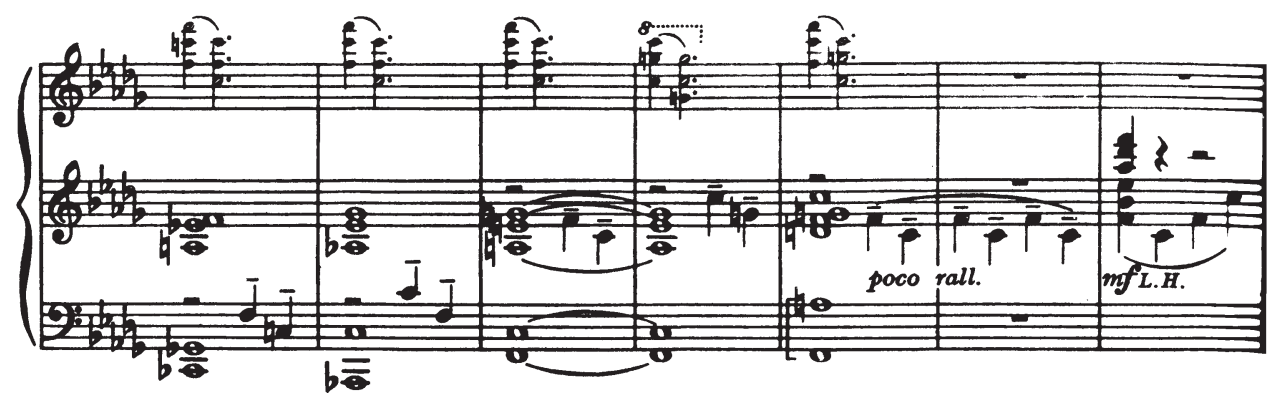

Beispiel 36: George Gershwin, Porgy and Bess, Vorspiel zu Akt 3, 446/4

Die Erweiterung des Major-Septakkordes mit der großen None und gleichzeitig der großen Sexte über dem Grundton wie in Beispiel 37 (T. 1, 1. bis 7. Achtel) ist selten.

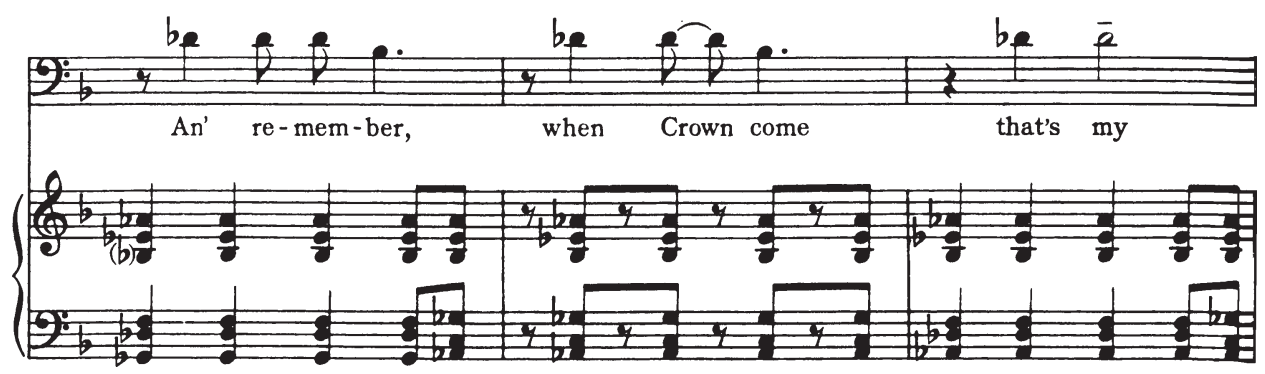

Beispiel 37: George Gershwin, Porgy and Bess, Akt 2, Szene 3 (»l loves you, Porgy«), 354/3

Sie wirkt wie eine Mischung aus einem Major-Nonakkord und einer Do-Pentatonik (s. u.). Der Klang kann ebenfalls in tonikaler oder subdominantischer Funktion auftreten und wird mit dem Kürzel 6/9 bezeichnet. Er findet sich in Beispiel 8 in T. 2, 1. Viertel (G6/9). 
Jazztypische Hinzufügungen zu einer Major-Septakkord-Basis sind außerdem noch die \#11 und die große 13, die der lydischen Skala angehören. Sie spielen in Gershwins Harmonik jedoch eine untergeordnete Rolle.

\section{MEHRKLÄNGE MIT MOLLBASIS}

Auch die Mehrklänge mit einer Mollbasis weisen eine geringe Vielfalt von Erweiterungsmöglichkeiten auf. Unterscheiden kann man hier zwischen zwei verschiedenen Septakkordtypen als Basis.

\subsection{Mollseptakkorde}

Der sehr gebräuchliche Mollseptakkord beinhaltet über dem Molldreiklang eine kleine Septime. Dieser Basisklang wird im Jazz üblicherweise mit der großen None erweitert. (Die kleine None ist aus klanglichen Gründen kein gängiger Optionston.) Beispiel 38 zeigt ein Moll-7/9-Voicing (T. 3, 1. Viertel): In engster Lage der Oberstimmentöne (aller Töne außer dem Bass) wäre es ein enges, terzgeschichtetes Voicing; durch die `drop-2 «Technik erhält man die ebenfalls sehr gängige und gut klingende gemischte Lage.

Bei Gershwin finden sich darüber hinaus viele Mollseptakkorde, die zwar nicht durch die None, aber durch höherliegende Optionstöne erweitert sind, nämlich die reine 11 und gelegentlich die große 13. Alle bisher genannten Optionstöne entstammen der dorischen Skala über dem jeweiligen Akkordgrundton.

Das oben gezeigte Beispiel 34 enthält über dem Grundton a (T. 1, 1. Hälfte) einen dorischen Moll-Sept-Undezimakkord. Beispiel 39 zeigt ein dorisches Total in Form des Akkords Dmin7/9/11/13 (T. 4).

\subsection{Moll-Major-Septakkorde}

Wesentlich seltener finden sich Molldreiklänge mit großer Septime. Beispiel 40 zeigt diesen nicht-diatonischen Klang (T. 1f). Der Ton $b$ in der Singstimme ist figurativ.

Im klassischen Jazz hat der Moll-Major-Septakkord als tonikaler Schlussklang eine gewisse Bedeutung; er kann auch mit der großen None erweitert sein. (Umgangssprachlich wird diese Art von Nonakkord auch als ’James-Bond-Klang، bezeichnet, da er den Schlussakkord im >Bond-Thema bildet.)

\subsection{Moll-Dreiklänge mit anderen Erweiterungen}

In Porgy and Bess finden sich an vielen Stellen Molldreiklänge ohne Septime, deren Erweiterungen aus der dorischen Skala über dem entsprechenden Grundton stammen. Der Basisklang ist in diesen Fällen der Molldreiklang mit der großen (dorischen) Sexte über dem Grundton. Das wohl prominenteste Beispiel hierfür bietet die Arie »Summertime» (Beispiel 41). Über den Basstönen $h$ und cis erklingt parallel verschoben (also als kurze 


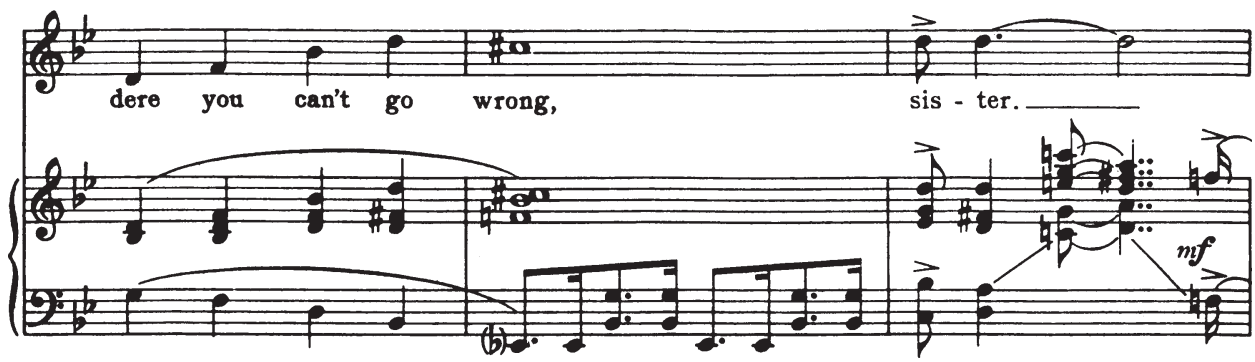

Beispiel 38: George Gershwin, Porgy and Bess, Akt 3, Szene 2 (»There's a boat dat's leavin' soon «), 498/3

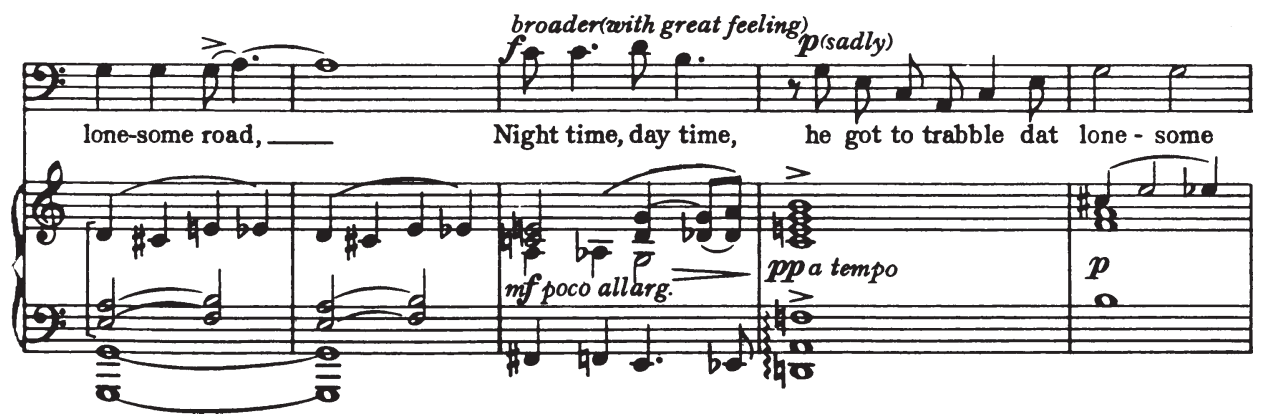

Beispiel 39: George Gershwin, Porgy and Bess, Akt 1, Szene 1 (»No, no, brudder«), 51/4

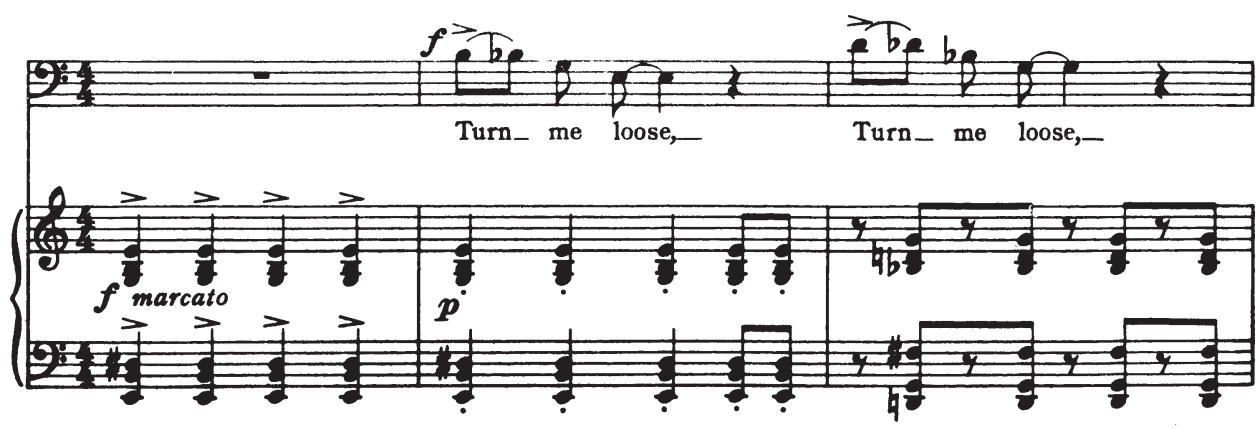

Beispiel 40: George Gershwin, Porgy and Bess, Akt 3, Szene 2 (»Turn me loose«), 491/3

reale Mixtur) jeweils ein Molldreiklang mit großer Sexte (T. $4 \mathrm{f}$.). Wegen der fehlenden Septime spricht man nicht von einem Tredezimklang; die Abkürzung beispielsweise für den ersten Akkord lautet als englisches Symbol nicht Bmin13, sondern Bmin6. 


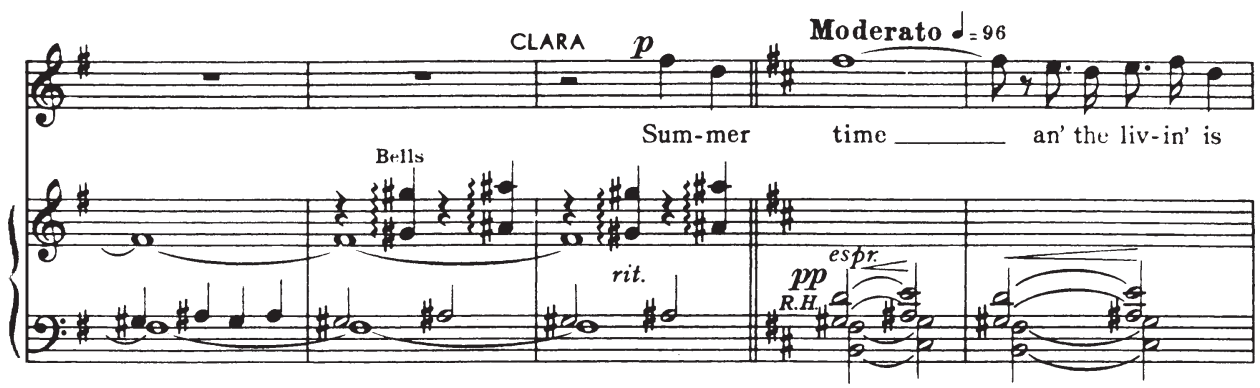

Beispiel 41: George Gershwin, Porgy and Bess, Akt 1, Szene 1 (»Summertime«), 15/1

Beispiel 42 zeigt einen Fünfklang, der außer der großen Sexte noch die große None über dem Grundton enthält (u.a. T. 1, 1. Viertel). Dieser ebenfalls sehr gebräuchliche Klang wechselt figurativ mit einem Moll-Septakkord; insgesamt erklingen 6 von den 7 Tönen der es-dorischen Skala.

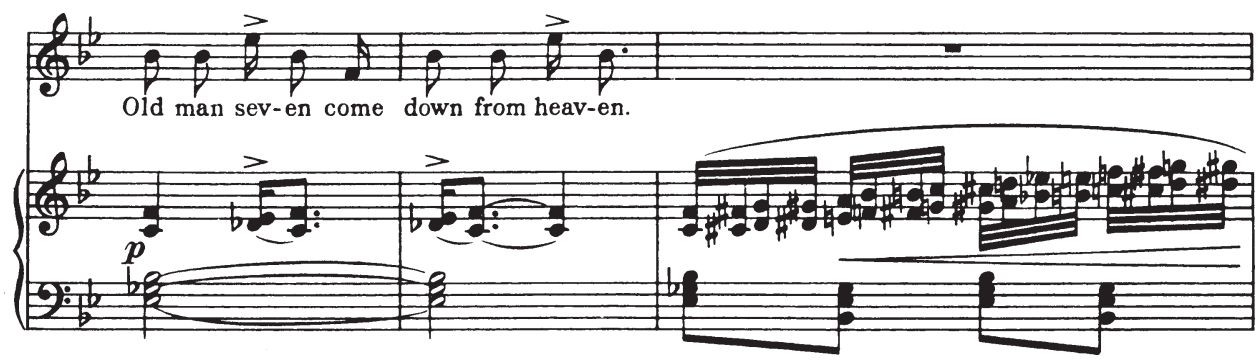

Beispiel 42: George Gershwin, Porgy and Bess, Akt 1, Szene 1 (»Fade me«), 63/2

In Beispiel 43 ist der vergleichbare 6/9-Klang zusätzlich mit der reinen (dorischen) 11 erweitert (T. 2). Wiederum erklingen 6 von 7 Skalentönen.

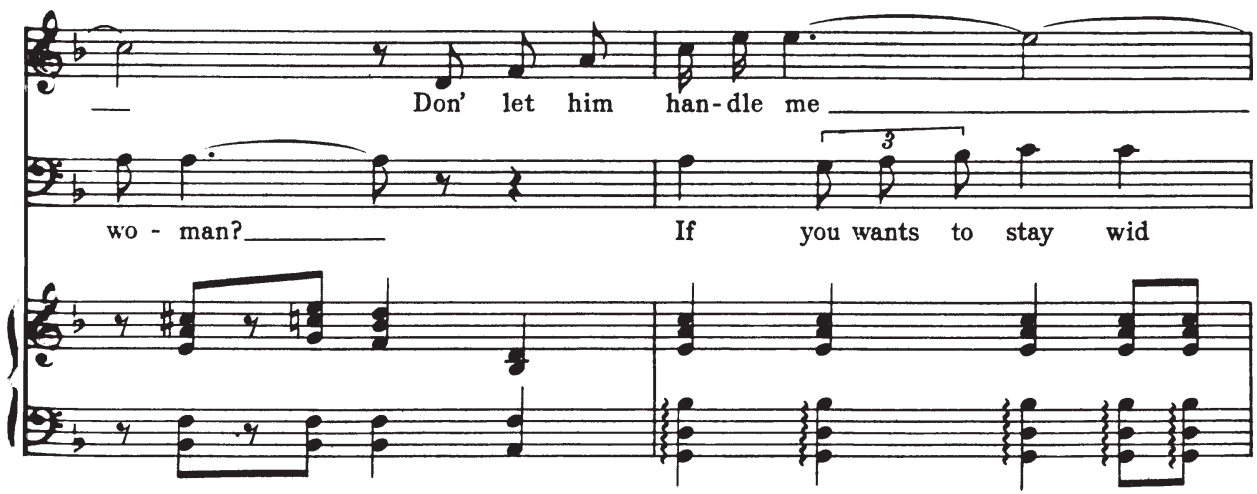

Beispiel 43: George Gershwin, Porgy and Bess, Akt 2, Szene 3 (»l loves you, Porgy«), 355/3 


\section{MEHRKLÄNGE MIT HALBVERMINDERTER BASIS}

Halbverminderte Septakkorde mit höherliegenden Optionstönen sind selten und werden hier nur der Vollständigkeit halber erwähnt. Beispiel 44 zeigt im ersten Akkord über dem Grundton fis einen halbverminderten Septakkord mit der reinen 11 als Optionston. Durch die anschließende Verschiebung des Klangs entsteht eine figurierte reale Mixtur.

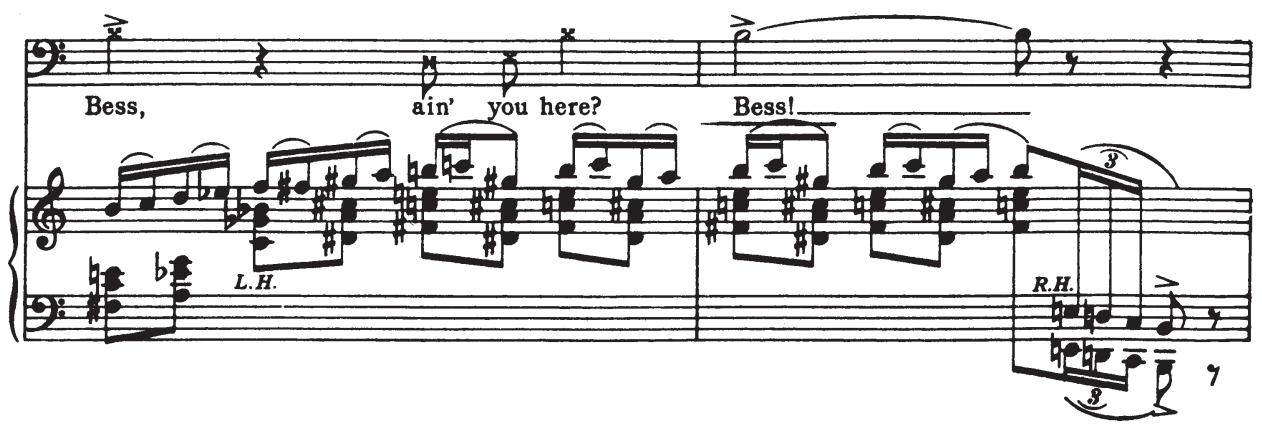

Beispiel 44: George Gershwin, Porgy and Bess, Akt 3, Szene 3 (»Bess, ain’t you here?«), 536/4

In der Jazz-Symbolik ist für den ersten Klang die Abkürzung F\#min7(b5)11 üblich. Verwirrend ist dabei, dass die Abkürzung des halbverminderten Septakkords zunächst die gleiche wie für einen Mollseptimakkord ist (F\#min7) und erst anschließend die verminderte (statt der reinen) Quinte chiffriert wird. Dies führt nicht selten zu der unsinnigen Übersetzung `Moll-verminderter Klang ${ }^{12}{ }^{2}$

\section{PENTATONISCHE KLÄNGE}

Pentatonische Mehrklänge spielen in der Jazz-Harmonik und speziell bei Gershwin eine große Rolle. Die Benennung der verschiedenen Ausprägungen erfolgt in Anlehnung an die relative Solmisation: Die wohl geläufigste Pentatonik mit den Tönen 1-2-3-5-6 der Durskala, solmisatorisch ausgedrückt Do-Re-Mi-Sol-La, wird über jedem Grundton als Do-Pentatonik bezeichnet (also C-Do-Pentatonik, G-Do-Pentatonik etc.). ${ }^{13}$ Jede Parallelskala hierzu wird ebenfalls mit dem absoluten Tonnamen und der relativen Solmisationssilbe bezeichnet. So können die pentatonischen Klänge benannt und im Hinblick auf ihre Stabilität sowie ihr Vorkommen auf bestimmten Stufen eingeordnet werden. Die Abbildung zeigt die C-Do-Pentatonik und ihre Parallelskalen (also D-Re-Pentatonik, E-Mi-Pentatonik, G-So-Pentatonik und A-La-Pentatonik) sowie als weiteres Beispiel die

12 Weitere Optionstöne sind denkbar, für Porgy and Bess jedoch nicht relevant: So wird im klassischen Jazz oft die große None über einem halbverminderten Klang ergänzt, zuweilen auch die kleine Tredezime. Insgesamt werden diejenigen Optionstöne verwendet, die einer Variante der lokrischen Skala mit der großen Sekunde über dem Grundton entstammen (১Locrian $\sharp 2$ «). Hierbei handelt es sich um eine Parallelskala der akustischen Skala.

13 Vgl. ebd., 224. 
F-Do-Pentatonik (ebenfalls mit Parallelskalen, also G-Re-Pentatonik, A-Mi-Pentatonik, C-So-Pentatonik, D-La-Pentatonik). ${ }^{14}$

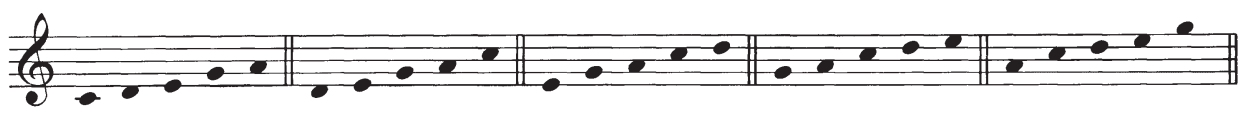
do re mi so la re mi so la do $\mathrm{mi}$ so la do re so la do re $\mathrm{mi}$ la do re $\mathrm{mi}$ so

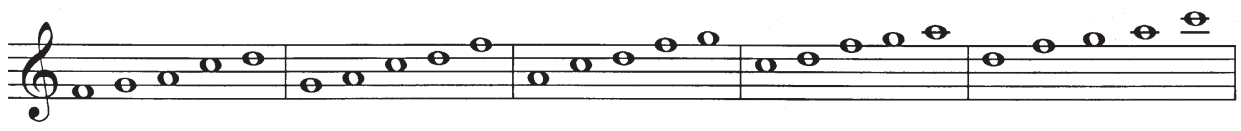

Beispiel 45: Do-Pentatoniken und ihre Parallelskalen ${ }^{15}$

So kann man die bekannte Akkordfolge aus dem ersten Chor von Porgy and Bess als ein Pendel zwischen einer G-Do-Pentatonik und einer C-So-Pentatonik verstehen. Bemerkenswert ist hier weiterhin, dass die rechte Hand in realen Mixturen fortschreitet, denn die drei Töne werden jeweils im Ganztonabstand versetzt. Aufgrund der geringen Anzahl der Töne ist diese Mixtur gleichzeitig tonal in G-Mixolydisch, derjenigen Diatonik, in der sich sämtliche Takte ausschließlich bewegen und die mittels der beiden erwähnten Pentatoniken vollständig mit allen sieben Tönen dargestellt wird.

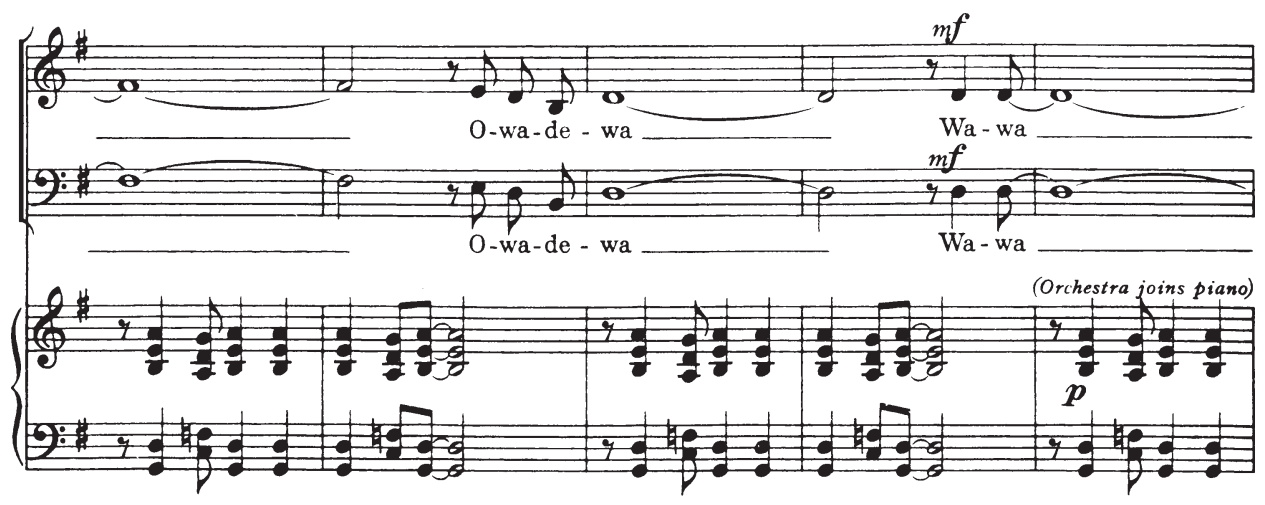

Beispiel 46: George Gershwin, Porgy and Bess, Akt 1, Szene 1 (»Wawa Dooda«), 10/2

\subsection{Do-Pentatoniken}

Die Do-Pentatonik erscheint meist auf der I. Stufe von Dur-Tonarten. Sie ist identisch mit dem jazztypischen Dur-6/9-Klang, der oft als (Schluss-)Tonika verwendet wird. Wie bereits erwähnt, zeigt Beispiel 8 in T. 2, 1. Viertel, eine tonikale G-Do-Pentatonik.

14 Vgl. ebd., 245.

15 Vgl. ebd., 245. 
Beispiel 47 enthält Do-Pentatoniken auch auf anderen Stufen: Prinzipiell kommt jede Stufe in Frage, die nicht-dominantisch besetzt werden kann und eine Dur-Dreiklangsbasis hat. In Beispiel 47 erklingt im ersten Takt zuerst eine C-Do-Pentatonik (I. Stufe), in der zweiten Takthälfte eine As-Do-Pentatonik (bVI. Stufe), danach eine F-Do-Pentatonik (IV. Stufe). Die drei pentatonischen Klänge sind dabei in verschiedenen Lagen ausgeprägt: Bei der C-Do-Pentatonik liegt im unfigurierten Akkordgriff der Ton g oben (Quintlage), die As-Do-Pentatonik erscheint in Sextlage, die F-Do-Pentatonik in Nonlage. Die Abstände der Töne erlauben, im ersten Akkord von einer weiten Lage zu sprechen, beim zweiten von einer engen und beim dritten von einer gemischten Lage. Alle diese Ausprägungen sind im Jazz-Klaviersatz üblich.

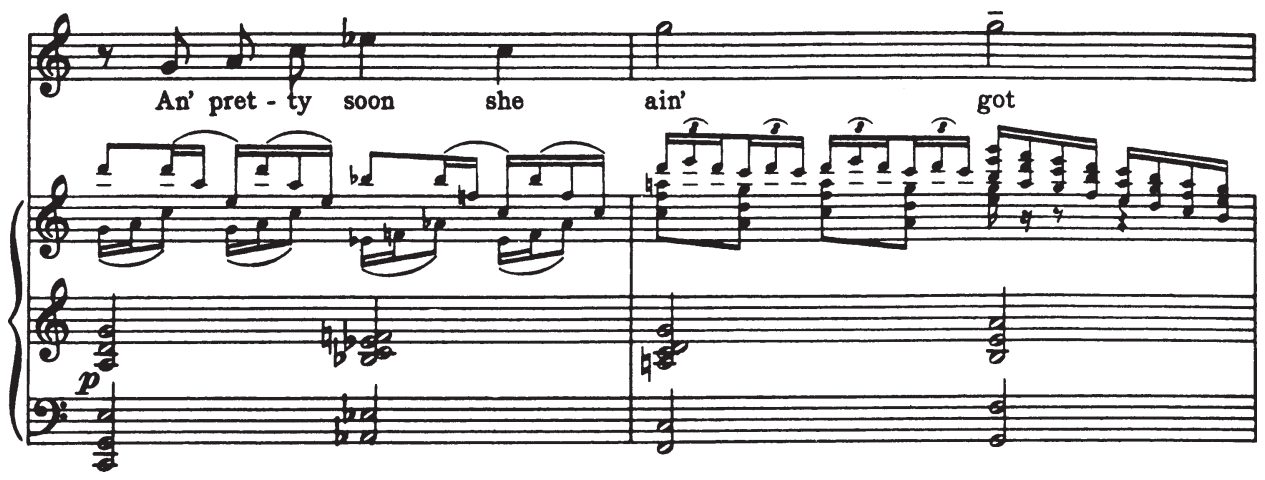

Beispiel 47: George Gershwin, Porgy and Bess, Akt 3, Szene 1 (»Crown, Crown«), 458/1

\subsection{Re-Pentatoniken}

Spielt man über dem Grundton der V. Stufe in Dur die Töne einer Re-Pentatonik, entsteht ein Klang, der mit einem Dominantseptnonakkord einschließlich Quartvorhalt identisch ist. Abgekürzt erscheint ein solcher Klang in Jazz-Standards als Dsus7/9 (oder einfach Dsus). Das Kürzel ssusı steht für ssuspendedı, also svorgehalten`.

In Beispiel 48 ist die Vorhaltswirkung besonders deutlich, weil die Quarte über dem Grundton jeweils in die große Terz aufgelöst wird.

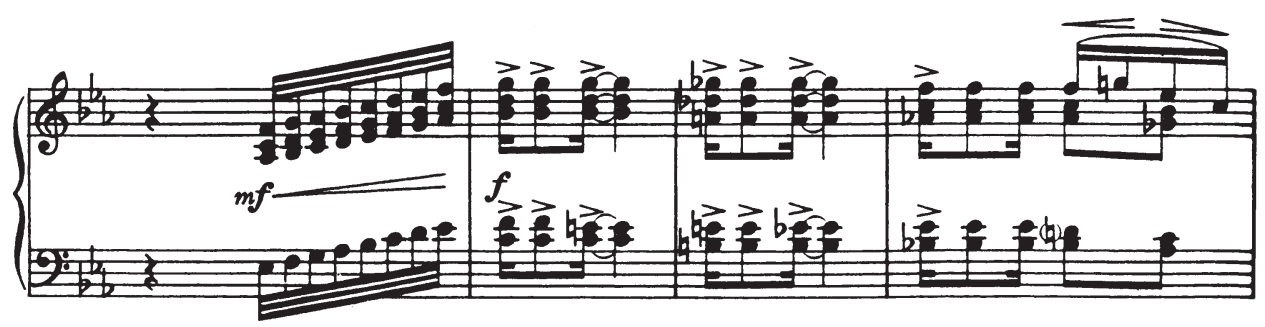

Beispiel 48: George Gershwin, Porgy and Bess, Akt 1, Szene 2 (»Gone, gone, gone«), 122 (ohne Chorstimmen) 
Zunächst erklingt eine C-Re-Pentatonik mit Auflösung zu C7/9 (T. 2), danach das Gleiche über $h$ und schließlich noch einmal über $b$. Insgesamt entsteht also eine reale Sequenz mit Versetzung der einzelnen Sequenzmodelle im Halbtonabstand.

Eine andere, weitaus seltenere Weise, Re-Pentatoniken zu verwenden, zeigt Beispiel 49. Der erste Klang ist eine G-Re-Pentatonik (im F-Dur-Kontext also auf der II. Stufe). Dieser Klang wirkt ebenfalls als Vorhaltsklang; er löst sich noch im gleichen Takt in einen G min7-Akkord auf (2. Takthälfte).

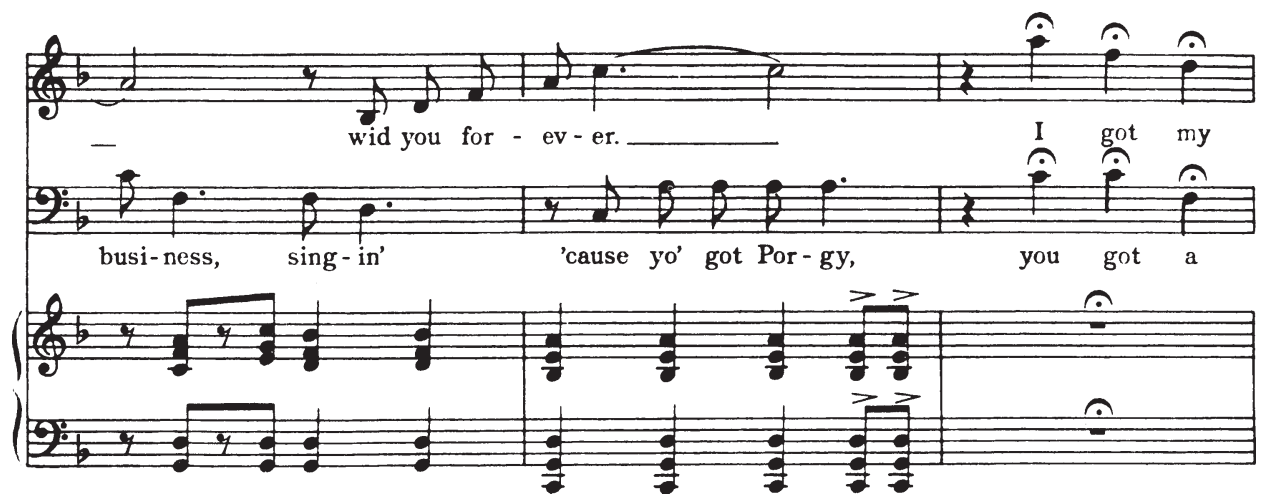

Beispiel 49: George Gershwin, Porgy and Bess, Akt 2, Szene 3 (»l loves you, Porgy«), 357/1

\subsection{La-Pentatoniken}

Beispiel 50 steht ebenfalls in F-Dur. Der Klang auf dem 4. Viertel des 1. Taktes kann als A-La-Pentatonik bezeichnet werden. Das Jazz-Symbol für diesen Klang wäre Amin7/11.

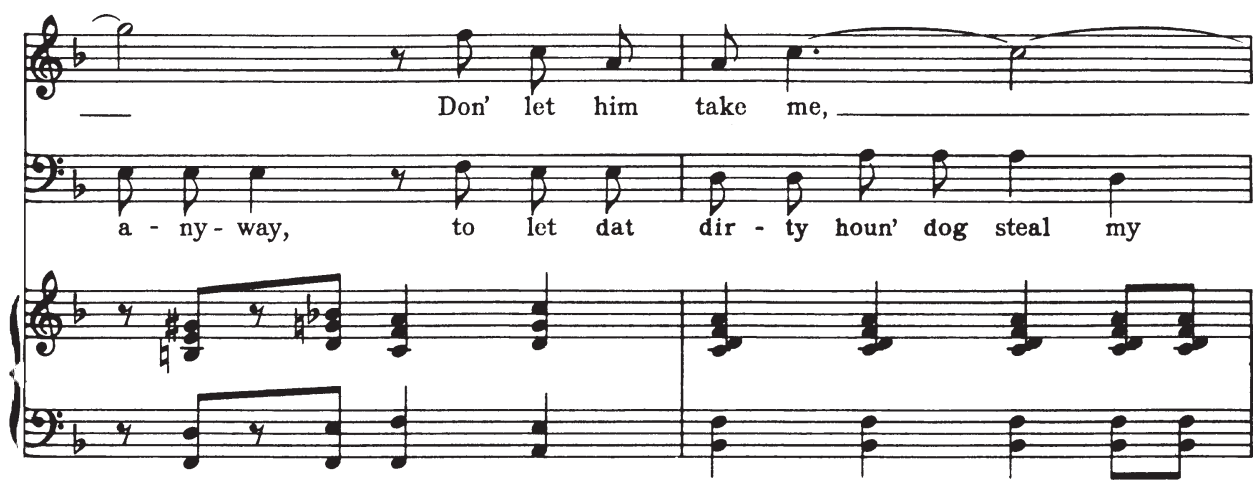

Beispiel 50: George Gershwin, Porgy and Bess, Akt 2, Szene 3 (»l loves you, Porgy«), 355/2

Solche Mollseptakkorde mit der sehr gebräuchlichen Hinzufügung der reinen Undezime können in Dur-Tonarten auf der II., III. und VI. Stufe vorkommen, in Moll-Tonarten auf der I. und IV. Stufe (sowie auf der II. Stufe, falls der Tonvorrat des melodischen Moll ver- 
wendet wird). Wie bereits unter 3.1 erwähnt, enthält Beispiel 34 diese Klangbildung auf der II. Stufe in der Tonart G-Dur.

\subsection{So- und Mi-Pentatoniken}

So-Pentatoniken kommen wesentlich seltener vor als die drei bisher besprochenen Pentatonik-Arten. Für sie gibt es kein Äquivalent in der Jazz-Symbolik. (Zwar wäre es möglich, den Klang als Dur-6/9-Akkord mit Quinte im Bass zu bestimmen, doch ist dies nicht üblich.)

Beispiel 51 zeigt eine As-So-Pentatonik im Rahmen einer Orgelpunkt-Wirkung (2. Takt, 3. Viertel). Der Klang wirkt wie eine IV. Stufe über dem Quintorgelpunkt.

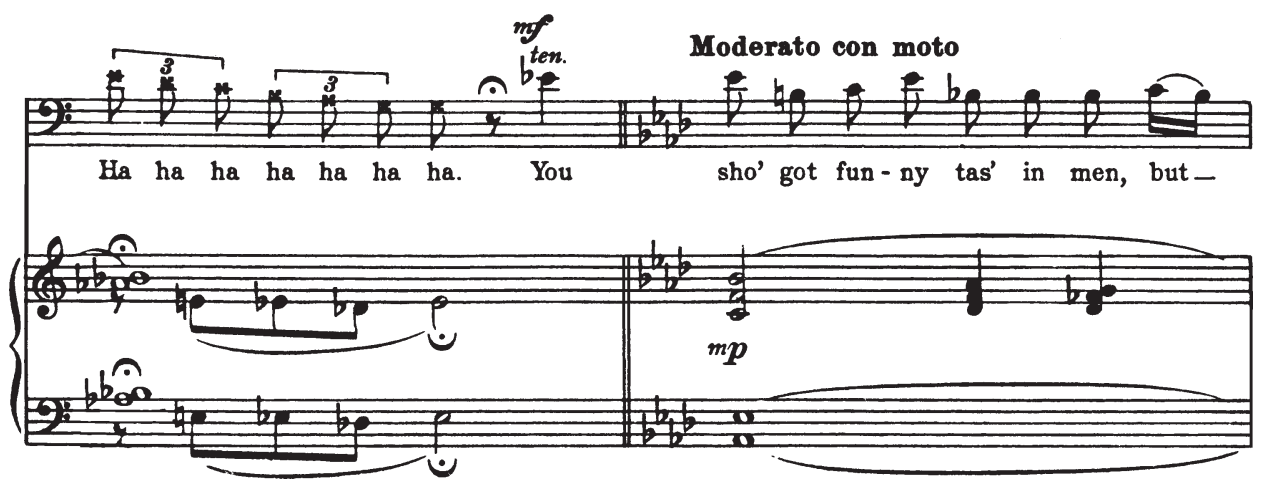

Beispiel 51: George Gershwin, Porgy and Bess, Akt 2, Szene 2 (»You sho’ got funny tas` in men«), $307 / 4$

Auch in Beispiel 37 findet sich eine As-So-Pentatonik (T. 3). Hier entsteht im Rahmen der aktuellen Grundtonart Des-Dur der Klangeindruck eines tonikalen Akkords über dominantischem Orgelpunkt.

Die seltenen Mi-Pentatoniken wirken ebenfalls oft wie Umkehrungen von tonikalen Pentatoniken. Für Mi-Pentatoniken gibt es, genauso wie für So-Pentatoniken, kein jazztypisches Symbol. (Zwar ließe sich von Dur-6/9-Akkorden mit Terz im Bass sprechen, doch ist diese Bestimmung ungebräuchlich.)

In Beispiel 52 sieht man als ersten Akkord von T. 4 eine G-Mi-Pentatonik (1. Achtel). Die ersten drei Achtel des Takts enthalten ausschließlich Töne von Es-Dur, daher klingt die eröffnende Mi-Pentatonik tonikal.

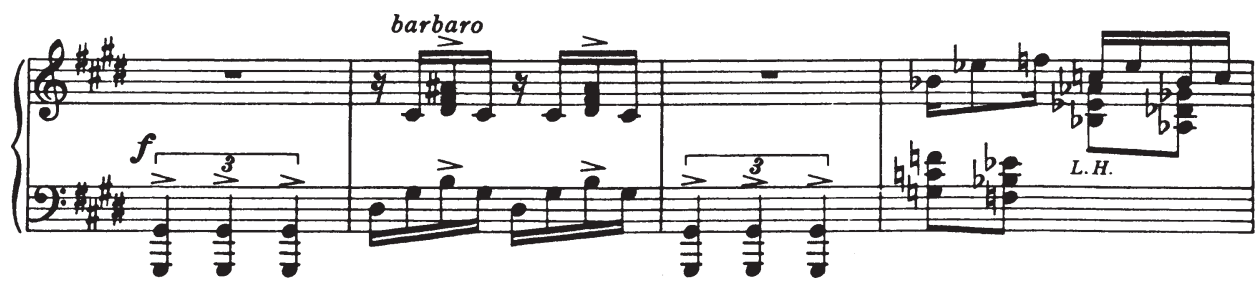

Beispiel 52: George Gershwin, Porgy and Bess, Akt 3, Szene 3 (»La, la, la«), 518/2 


\section{KLAVIERPRAKTISCHE ÜBUNGEN}

Um die genannten Skalen und Akkorde für eigene Jazz-Improvisationen verfügbar zu machen, kann man für verschiedene gängige Klaviergriffweisen systematische Übungsmodelle entwerfen.

\subsection{Skalenübungen}

Auf jedem Grundton sollen die akustische, die alterierte und außerdem jede mögliche pentatonische Skala gespielt werden.

Für die pentatonischen Skalen bedeutet dies: Nacheinander können innerhalb eines pentatonischen Tonvorrats (z. B. `Schwarze-Tasten-Pentatonikı) sämtliche Parallelskalen gespielt werden (also Fis-Do, Gis-Re, Ais-Mi, Cis-So, Dis-La), was nicht in jedem pentatonischen Tonvorrat so leicht zu bewerkstelligen ist wie im Falle der Schwarze-TastenPentatonik.

Ferner kann von einem festgelegten Grundton aus jede der fünf möglichen Pentatoniken gespielt werden, also beispielsweise nacheinander C-Do (Töne $c-d-e-g-a), C-R e$ (Töne $c-d-f-g-b), C-M i(T o ̈ n e ~ c-e s-f-a s-b), C-S o(T o ̈ n e ~ c-d-f-g-a), C$-La (Töne $c-e s-f-g-b$ ).

\subsection{Akkordübungen in enger Lage}

\section{Septnonakkorde}

Bereits mit Septnonakkorden kann man ein stilistisch taugliches Comping vieler JazzStandards spielen. Um sich die Akkorde spieltechnisch zu erschließen, empfiehlt sich zu Beginn folgendes Vorgehen:

- Man greift in der linken Hand ausschließlich den Basston der Akkorde (der für die ersten Übungen stets dem Grundton entsprechen sollte).

- In der rechten Hand greift man zuerst nur den Dreiklang, und zwar entweder in Oktavlage (Töne 3-5-8) oder in Quintlage (Töne 1-3-5; die 1 kann auch als 8 bezeichnet werden, also 8-3-5).

- Ordnet man den Tönen diese Zahlen zu, kann man nun leicht den Nonakkord finden: Die 7 findet sich immer im Sekundabstand links neben der 8; die 9 findet sich immer im Sekundabstand rechts neben der 8. Der Oktavton über dem Grundton wird also sozusagen nach links und rechts aufgespalten.

Man muss jeweils entscheiden, ob die große 7 (nur für Major-Septnonakkorde) oder die kleine 7 (für alle anderen Septnonakkorde) gebraucht wird. Ebenso muss man darauf achten, die kleine 9 (nur bei Dominant-Septakkorden mit kleiner None) von der großen None (bei allen anderen Septnonakkorde) zu unterscheiden.

Geht man bei dieser Übung von der Quintlage des Dreiklangs aus, erhält man dasjenige Voicing, bei der die Septime der tiefste Ton der rechten Hand ist. Bei der Oktavlage des Dreiklangs erhält man das andere gängige Voicing: Hier ist der Terzton der tiefste Ton 
der rechten Hand. (Möchte man einen Akkord des Typs 7/ \#9 greifen, kann man ebenso verfahren. Allerdings lässt man dann aus klanglichen Gründen oft die Quinte weg.)

\section{Non-Tredezimakkorde}

Für das (für den Anfänger oft mühselige) Auffinden von Non-Tredezimakkorden kann man in der beschriebenen Weise zunächst den fraglichen Nonakkord suchen. Am häufigsten wird man eine dominantische Septakkordbasis mit großer None, am zweithäufigsten eine dominantische Septakkordbasis mit kleiner None verwenden.

Ausgehend von diesem Griff (links Grundton, rechts die Töne 3-5-7-9) kann man nun anstelle der 5 die 6 spielen (sie entspricht ja der oktavreduzierten 13), also den rechts sekundweise benachbarten Ton. Man muss dann nur noch beachten, ob die große oder die kleine 13 gefragt ist.

(Theoretisch lassen sich Non-Undezimakkorde auf die gleiche Weise auffinden. Allerdings ergeben sich dabei nur für den akustischen Non-Undezimakkord halbwegs gängige Voicings. Für den Moll-Undezimakkord - den dorischen - sollte man die Töne terzgeschichtet, also in der Anordnung 1-3-5-7-9-11 greifen. Dabei muss also die linke Hand außer dem Grundton mindestens einen weiteren Akkordton greifen.)

\section{'Left hand voicings}

In der rechten Hand entstehen jeweils viertönige, grundtonlose Griffe, wenn man so verfährt, wie es in den vorigen Abschnitten erläutert wurde. Spielt man diese Griffe in der linken statt in der rechten Hand, so erhält man die gängigsten der sogenannten ıleft hand voicings . Sie werden unter anderem dann benutzt, wenn ein anderes Instrument eine grundtonhaltige Basslinie spielt. Der Pianist hat so die rechte Hand frei, um Solo-Linien zu improvisieren. Allerdings bedeutet es eine große Umstellung, die viertönigen Voicings in die linke Hand sumzulegen

\section{Undezim- und Tredezimakkorde ohne None}

Spielt man ein sehr einfaches Comping auf Septakkordbasis, also ohne Nonen, kann man dabei gelegentlich auch Undezim- oder Tredezimakkorde ohne None einbauen. Man verfährt dann in ähnlicher Weise wie oben beschrieben: Zunächst greift man einen (in der rechten Hand dreitönigen) Septakkord so, dass einer der Funktionstöne (am besten die Septime) der tiefste Ton der rechten Hand ist. Dann verschiebt man den `QuintFinger sekundweise nach links zur passenden 11 (bei dominantischer Basis übermäßige 11, bei Moll- oder halbverminderter Basis reine 11) oder sekundweise nach rechts zur passenden 13 (bei dominantischer Basis groß oder klein, bei jeder anderen Basis groß).

\section{3 Übungen zu >drop-2-voicings`}

Die unter 6.2 beschriebenen nonenhaltigen Akkordtypen, bei denen man viertönige Griffe in der rechten Hand verwendet, können gut in satt klingende sdrop-2-voicings umgewandelt werden. Folgende zwei Arbeitsschritte empfehlen sich: 
- Man legt in der linken Hand den Basston (= Grundton) des Akkords so tief, dass zwischen den beiden Daumen mindestens eine Septime Abstand ist. Allerdings sollte dabei der Ton $c 0$ nicht unterschritten werden.

- Man ermittelt aus dem viertönigen Griff der rechten Hand den zweiten Ton von oben und lässt ihn um eine Oktave nach unten fallen, wo er von der linken Hand abgegriffen wird. In der linken Hand entsteht dadurch ein Griff aus Grundton plus Terz oder aus Grundton plus Septime.

\subsection{Pentatonische Akkorde}

Die einfachste Griffweise pentatonischer Akkorde besteht darin, in der linken Hand den Grundton und in der rechten Hand in engster Lage die vier übrigen Töne zu spielen. Wegen der entstehenden Sekundreibungen klingen diese Griffe nicht immer gut.

\section{Do-Pentatoniken}

Wie oben erklärt, entspricht die Do-Pentatonik der häufigen tonikalen Akkordausprägung Dur 6/9. Greift man in der linken Hand den Grundton und in der rechten Hand die vier übrigen Töne in enger Lage, empfehlen sich vor allem diejenigen Griffe, bei denen der tiefste Ton der rechten Hand der Terz oder der Quinte des Akkords entspricht. Dadurch ergibt sich zwischen dem Grundton und dem nächsthöheren Ton ein stabiles Akkordfundament. Die anderen beiden Griffe (None oder Sexte als tiefster Ton der rechten Hand) wirken weniger stabil.

Gut klingende weite Lagen entstehen über dem genannten stabilen Akkordfundament (Grundton plus Terz oder Grundton plus Quinte, am besten dann beides in der linken Hand gespielt), wenn man darüber eine quartige Auftragung der Töne wählt. Im Falle der C-Do-Pentatonik ergeben sich etwa die Varianten $c-e-a-d-g$ oder $c-g-e-a-d$.

\section{Re-Pentatoniken}

Die Re-Pentatonik ist eine gut klingende Umsetzung des Akkordsymbols sus7/9. Im Hinblick auf eine mögliche Auflösung in einen Nonakkord empfehlen sich hier vor allem diejenigen Griffe, bei denen der tiefste Ton der rechten Hand der Quarte oder der Septime über dem Grundton entspricht. Bei einer Auflösung der Quarte zur Terz entstehen dann die unter 6.2.1 beschriebenen Voicings. So ergeben sich etwa für die C-Re-Pentatonik (Csus7/9) folgende Auflösungen:

- Töne $c-f-g-b-d$; mögliche Auflösung zu c-e-g-b-d.

- Töne $c-b-d-f$-g; mögliche Auflösung zu c-b-d-e-g.

Die beiden genannten voicings der Re-Pentatonik lassen sich - wegen des Zusammenhangs mit den Nonakkorden - auch per `drop-2«-Technik zu gut klingenden weiten Lagen umwandeln. 


\section{Andere Pentatoniken}

Von den anderen drei Pentatoniken (La, So und Mi) lässt sich lediglich die La-Pentatonik als jazztypischer Akkord in einem Symbol umsetzen, nämlich min7/9/11. Für diesen Akkord bietet sich allerdings nur die oben erwähnte terzgeschichtete Griffweise an (z. B. Cmin7/9/11: von unten nach oben mit den Tönen $c$-es-g- $b-d-f)$. Engere Lagen sind ungebräuchlich.

So- und Mi-Pentatoniken sind nur im Rahmen von nicht-grundstelligen Akkorden relevant und so selten, dass sie zunächst nicht weiter berücksichtigt werden müssen.

\subsection{Reale Mixturen}

Die verschiedenen Akkordtypen lassen sich gut üben indem man sie in realen Mixturen spielt. Am einfachsten ist die chromatische Verschiebung, die freilich dazu verführt, einfach jeden Finger gedankenlos zu verschieben. Die ganztönige Verschiebung fällt meist schwerer. Noch effektiver ist die Verschiebung in klein- oder großterzigen Abständen.

Beim Spielen realer Mixturen wird zudem das Gehör geschult: Der Spieler muss bei jedem neuen Grundton beurteilen, ob der verschobene Akkord von den enthaltenen Intervallverhältnissen her genauso klingt wie der jeweils vorhergehende.

Die beschriebenen Akkordtypen sind nicht nur typisch für Gershwins Harmonik, sondern finden sich sehr häufig auch in jazzorientierten (Klavier-)Sätzen anderer Komponisten. Ihre Systematisierung, begriffliche Erfassung und spielpraktische Einübung erleichtert das lesende Auffinden und hörende Wiedererkennen dieser Klangtypen und bildet die Voraussetzung ebenso für die Analyse wie für Improvisations- und Kompositionsversuche in den entsprechenden Stilen.

\section{Noten}

Gershwin, George (1935), Porgy and Bess, Klavierauszug, hg. von Albert Sirmay, New York: Gershwin Publishing Corporation/Chappell.

\section{Literatur}

Gárdonyi, Zsolt / Hubert Nordhoff (2002), Harmonik, 2. Aufl., Wolfenbüttel: Möseler. Mark Levine (1992), Das Jazz Piano Buch, Rottenburg: Advance Music. 